Bell-Plesset effects for an accelerating interface with contiguous density gradients

P. Amendt

December 22, 2005

Physics of Plasmas 
This document was prepared as an account of work sponsored by an agency of the United States Government. Neither the United States Government nor the University of California nor any of their employees, makes any warranty, express or implied, or assumes any legal liability or responsibility for the accuracy, completeness, or usefulness of any information, apparatus, product, or process disclosed, or represents that its use would not infringe privately owned rights. Reference herein to any specific commercial product, process, or service by trade name, trademark, manufacturer, or otherwise, does not necessarily constitute or imply its endorsement, recommendation, or favoring by the United States Government or the University of California. The views and opinions of authors expressed herein do not necessarily state or reflect those of the United States Government or the University of California, and shall not be used for advertising or product endorsement purposes. 


\title{
Bell-Plesset effects for an accelerating interface with contiguous density gradients
}

\author{
Peter Amendt \\ Lawrence Livermore National Laboratory, University of California, Livermore, CA \\ 94551
}

\begin{abstract}
A Plesset-type treatment [J. Appl. Phys. 25, 96 (1954)] is used to assess the effects of contiguous density gradients at an accelerating spherical classical interface on Rayleigh-Taylor and Bell-Plesset perturbation growth. Analytic expressions are obtained that describe enhanced Rayleigh-Taylor instability growth from contiguous density gradients aligned with the acceleration and which increase the effective Atwood number of the perturbed interface. A new pathway for geometric amplification of surface perturbations on an accelerating interface with contiguous density gradients is identified. A resonance condition between the density-gradient scalelength and the radius of the interface is also predicted based on a linearized analysis of Bernoulli's equation, potentially leading to enhanced perturbation growth. Comparison of the analytic treatment with detailed two-dimensional single-mode growth-factor simulations shows good agreement for low-mode numbers where the effects of spherical geometry are most manifested.
\end{abstract}

\section{Introduction}

The problem of hydrodynamic stability control on accelerating interfaces is key to the successful demonstration of inertial confinement fusion (ICF) [1]. In this scheme a low-Z capsule containing deuterium-tritium (DT) fuel is ablatively driven by $\mathrm{x}$ rays in the indirect-drive (hohlraum) configuration [2], or by $\approx 1 / 3 \mu \mathrm{m}$ laser light in the direct-drive scheme [3]. As the compressed fuel responds to the imploding shell, the interface between the fuel and pusher decelerates and becomes Rayleigh-Taylor (RT) unstable. In cryogenic ICF schemes the pusher consists of DT ice, and the fuel-pusher interface is no longer localized to a surface but over a region with a finite density gradient. Much analytical and computational effort [4-8] as well as experimental work [9-10] has established that the presence of finite density gradients has a stabilizing effect on RT instability, increasing as the perturbation wavelength decreases in relation to the gradient scalelength. However, this stabilizing effect does not hold true for the DT ice-ablator interface in indirect-drive approaches where a material and density $(\rho)$ mismatch persist 
in the fluid limit. On this interface near classical RT growth can be expected: $\gamma_{R T, c}=\sqrt{A t \cdot k g}$, where $A t=\left(\rho_{2}-\rho_{1}\right) /\left(\rho_{2}+\rho_{1}\right)$ is the Atwood number between the outer ("2") and inner ("1") fluids, $g$ is the acceleration, $k$ is the perturbation wavenumber and $A t \cdot g>0$. A similar instability scenario applies to non-cryogenic double-shell ignition schemes [11]. For this proposed type of target, the high- $Z$ inner shell contains the room-temperature, high-pressure DT fuel and is generally characterized by a highAtwood number inner interface [12]. The same holds true in studies of cavitating bubbles in laser medicine [13] and sonoluminescence research [14] where the liquid-vapor interface is classically RT unstable.

In all these physical systems one or both sides of the classically RT unstable interface often have a contiguous nonzero density gradient. For example in the case of an imploding compressible shell, self-consistent interior pressure and density profiles are necessary to maintain a uniform acceleration of the shell [2]. For a cavitating bubble, a density profile in the vapor will be established as the bubble successively expands and collapses. The question that this paper addresses is to what extent the presence of contiguous density profiles affects RT growth in a converging geometry with a finite Atwood number on a classical interface. A related and coupled issue is to what degree, if any, will Bell-Plesset (BP) geometric growth [15] be affected by contiguous density profiles. Previous work by Bernstein and Book [16] and Lezzo and Prosperetti [17] has considered the RT question, though only in a slab geometry. Physically, the effect of contiguous density profiles on a classical (discontinuous) interface $(A t \neq 0)$ should be manifested most at wavelengths on the order of the density-gradient scalelength or greater, not less as in the converse case of a finite density gradient on the interface [4-8]. In the latter case, the RT growth rate in the planar limit obeys [8]:

$$
\gamma_{R T}=\sqrt{\frac{A t \cdot k g}{1+A t \cdot k L_{\rho}}}
$$

where $L_{\rho}$ is the density-gradient scalelength $(d \ln \rho / d r)^{-1}$. For the example of contiguous density profiles in a spherical geometry, a slab approximation may not suffice since the behavior of the important low-order Legendre modes will likely be 
misrepresented. Thus, capturing the essential influence of contiguous density profiles on a classical interface in an imploding shell is best suited to a spherical treatment.

As a starting point we adopt a Plesset-type velocity potential framework [12] for describing the compressible fluid dynamics of an accelerating shell. The perturbed velocity potential $\delta \Psi$ will necessarily satisfy Poisson's equation $\left(\nabla^{2} \delta \Psi \neq 0\right)$ in the presence of contiguous density gradients, implying what is understood in the literature as a compressible perturbed fluid response. However, we will distinguish between two physical regimes for the perturbed fluid response under these conditions, depending on whether the fluid density can adjust to the perturbed interface over a RT growth time $\gamma_{R T, c}^{-1}$, or not. The representative timescale for adjustment of the perturbed density is the reciprocal of the Laplacian of the perturbed velocity potential. Both perturbed fluid responses will be compressible according to the traditional standard that $\nabla^{2} \delta \Psi$ be nonzero, but the former case will more intuitively reflect our notion of compressible response. Thus, we will label the first response as "compressible" and the latter as "incompressible" for this purpose.

In this paper we derive the RT dispersion relation and describe BP effects in the presence of contiguous density profiles on both sides of a discontinuous, accelerated spherical interface separating two compressible fluids [See Fig. (1)]. For the simple case of equal density-gradient scalelengths and identical polytropes on each side of such an interface, the RT dispersion relation in the planar limit is analytically obtained:

$$
\gamma_{R T}=\sqrt{A t \cdot k g \cdot\left(\frac{2 k\left|L_{\rho}\right|}{\sqrt{1+4 k^{2} L_{\rho}^{2}} \pm A t}\right)},
$$

where the plus (minus) sign denotes $L_{\rho}<0\left(L_{\rho}>0\right)$. The small wavelength limit recovers the classical RT growth rate, while for very long wavelengths (or low mode numbers in spherical geometry) the growth is strongly reduced by density-gradient stabilization. For moderately long wavelengths $1-A t<2 k^{2} L_{\rho}^{2} \ll 1$, Eq. (1b) recovers 
the familiar limit $\gamma_{R T}=\sqrt{g / L_{\rho}}$ with $L_{\rho}>0$. In ICF applications, thermal conduction from the nearly isobaric fuel to the imploding shell generally establishes a positive density gradient $\left(L_{\rho}>0\right)$, resulting in increased RT growth after deceleration onset. The maximum increase in RT growth occurs for wavenumbers in the vicinity of $\sqrt{1-A t^{2}} /\left(2 L_{\rho} A t\right)$, leading to an enhanced growth rate: $\gamma_{R T, c} \cdot\left(1-A t^{2}\right)^{-1 / 4}$. Physically, the increased perturbation growth results from an effectively larger Atwood number when averaged over a distance across the classical interface on the order of the wavelength $2 \pi / k$.

Analysis of Bernoulli's integral predicts the occurrence of two resonances between the density-gradient scalelength and the radius of the interface for sufficiently large contiguous density gradients. These resonances may generally lead to enhanced density fluctuations for an imploding compressible shell during acceleration.

The effect of contiguous density gradients on classical BP effects is studied. A new pathway for BP growth is shown for an accelerating shell with contiguous density gradients on the inner interface.

Direct comparison of the analytic model with two-dimensional (2-D) single-mode growth-factor simulations of an ICF capsule implosion is also made. Good agreement for the lowest-order modes where BP effects are important is found. For higher-order modes the effect of thermal transport along a perturbation wavelength in reducing classical RT growth becomes significant. A heuristic adaptation of the model to include thermal diffusivity is largely successful in suppressing classical RT growth to levels seen in the 2D simulations.

The paper is organized as follows. Section II develops analytic estimates for contiguous density-gradient scalelengths and derives the governing equations for BP and RT growth. Section III applies the model to some general cases of interest in ICF. A specific comparison of the model with simulated growth factors for an indirectly-driven capsule implosion is presented in Section IV. We summarize in Section V. 


\section{Analysis}

\section{a) Density-gradient scalelengths}

Figure (1) shows the geometry of the problem under consideration: an imploding shell or pusher of fluid type " 2 " compressing a fluid or fuel of type " 1 ". On either side of the interface at $r=R(t)$ we define a dimensionless parameter $\sigma_{i}(t) \equiv R / L_{\rho, i}$, where

$$
\left.L_{\rho, i} \equiv\left(d \ln \rho_{i, 0} / d r\right)^{-1}\right|_{r=R}
$$

is the local density-gradient scalelength at the interface. We introduce trial density profiles in the fuel and pusher regions:

$$
\rho_{i, 0}(r, t)=\bar{\rho}_{i, 0}(t) \cdot\left(\frac{r}{R}\right)^{\sigma_{i}}
$$

where $\bar{\rho}_{i, 0}(t)$ is the density of the unperturbed $i$-th material at the interface. This generic density profile has the preferred property that the planar limit recovers the exponential profile:

$$
\rho_{i, 0}=\lim _{\sigma_{i}, R \rightarrow \infty} \bar{\rho}_{i 0} \cdot\left(\frac{r}{R}\right)^{\sigma_{i}}=\lim _{\sigma_{i} \rightarrow \infty} \bar{\rho}_{i, 0} \cdot\left(1-\frac{x}{L_{\rho, i} \sigma_{i}}\right)^{\sigma_{i}}=\bar{\rho}_{i, 0} \cdot e^{-x / L_{\rho, i}}
$$

where $x \equiv R-r$. In this limit, $L_{\rho, i}$ for the density-gradient scalelength applies over the entire half-space, not just at the interface. For the simple case of an isothermal atmosphere one can readily show [16]:

$$
L_{\rho, i}=\frac{\left.C_{S, i}^{2}\right|_{r=R}}{g}
$$


where $C_{S, i}=\left.\sqrt{\left(\partial P_{i 0} / \partial \rho_{i 0}\right)}\right|_{s}$ is the adiabatic sound speed. For the spherical case, we refer to Eqs. $(2,3)$ to generally define $L_{\rho, i}(r)=L_{\rho, i}(R) \cdot r / R$ away from the interface. The importance of a radially varying, density-gradient scalelength is that the growth of long wavelength perturbation modes whose spatial extent is a significant fraction of the interface radius may be significantly affected. We address this issue further in Section IId.

In ICF applications, uniformly accelerating shells are typically considered for analytically assessing hydrodynamic stability [2]. For this class of equilibrium, we can deduce a relation between $\sigma_{i}$ and the polytropic index $\gamma_{i}$. Applying force balance in a comoving frame: $d P_{i, 0} / d r=-g \cdot \rho_{i, 0}$, and using a polytropic equation-of-state: $P_{i, 0} \rho_{i, 0}^{-\gamma_{i}}=C_{i}$, we obtain in place of Eq. (3):

$$
\rho_{i, 0}(r, t)=\bar{\rho}_{i, 0}(t) \cdot\left[1-\frac{g \cdot\left(\gamma_{i}-1\right)(r-R)}{C_{s, i}^{2}}\right]^{\frac{1}{\gamma_{i}-1}} .
$$

This density profile agrees with Eq. (3) only in the limit as $\gamma_{i} \rightarrow 1$, reducing to Eq. (4) in the end. Thus, standard planar treatments that use a uniformly accelerating shell will differ from Eq. (3) when $\gamma_{i}>1$, which defines the regime of interest in this work. Incidentally, we can also obtain a simple relation between $\sigma_{1}$ and $\sigma_{2}$ for this particular profile. By imposing pressure balance at $r=R$ and using Eq. (2) together with the definition of $\sigma_{i}$, we straightforwardly obtain:

$$
\frac{\sigma_{2}}{\sigma_{1}}=\frac{\bar{\rho}_{2,0}}{\bar{\rho}_{1,0}} \cdot \frac{\gamma_{1}}{\gamma_{2}} .
$$

Figure $2 \mathrm{a}$ shows examples from a radiation-hydrodynamic simulation of a proposed ignition double-shell design [11] for the shell density profile at several times following deceleration onset. Two classes of density-gradient scalelength are apparent. First, establishment of the isothermal atmospheric profile described above [See Eqs. (4-5)] is 
seen to occur in the outer half of the Au shell, particularly at later times relative to deceleration onset. By itself, this density profile would help suppress RT growth because the spatially averaged Atwood number decreases with increasing perturbation wavelength. However, a second density feature is evident near the inner surface of the shell. The associated density gradients are large and positive just outside the interface within the shell, potentially giving rise to increased instability growth. The origin of this second density feature is due to heat conduction from the hotter fuel to the shell and is independent of the sign of the shell acceleration. We can estimate the expected size of the density gradient as follows. Assuming Spitzer conductivity the thermal energy flux $Q$ from the fuel to the shell at the interface satisfies [18]: $\vec{Q}=S_{1} T_{1}^{5 / 2} \vec{\nabla} T_{1}=S_{2} T_{2}^{5 / 2} \vec{\nabla} T_{2}$, where

$$
S_{i} \approx-\frac{4}{\left(Z_{i}+3.3\right) e^{4} m_{e}^{1 / 2} \ln \Lambda_{i}}
$$

$Z_{i}$ is the ion charge state in region $i$ and $T_{i}$ is in units of eV. From Eq. (8a) we can readily form the ratio of the temperature-gradient scalelengths $L_{T, i} \equiv 1 /\left(d \ln T_{i} / d \ln r\right)$ at the interface:

$$
\frac{L_{T, 1}}{L_{T, 2}}=\frac{S_{1}}{S_{2}} \cdot\left[\frac{\bar{\rho}_{2,0}}{\bar{\rho}_{1,0}} \cdot \frac{Z_{2}+1}{Z_{1}+1} \cdot \frac{A_{1}}{A_{2}}\right]^{7 / 2},
$$

where ideal equations-of-state are assumed, $\ln \Lambda_{1} \approx \ln \Lambda_{2}$, and $A_{i}$ is the average number of nucleons per ion. At deceleration onset $\vec{\nabla} P_{i, 0} \approx 0$, and we find from the definition of $\sigma_{i}[$ See above Eq. (2)] and Eqs. (8a-b):

$$
\frac{\sigma_{2}}{\sigma_{1}} \approx\left(\frac{1+A t}{1-A t}\right)^{7 / 2}\left(\frac{Z_{2}+3.3}{Z_{1}+3.3}\right) \cdot\left[\frac{Z_{2}+1}{Z_{1}+1} \cdot \frac{A_{1}}{A_{2}}\right]^{7 / 2}
$$

For a gold pusher and DT fuel with $Z_{2} / Z_{1} \approx 25-50$ and $A t \approx 0.7-0.5$ after deceleration 
onset, Eq. (8c) gives $\sigma_{2} / \sigma_{1}=L_{\rho, 1} / L_{\rho, 2} \approx 10-30$. Furthermore, with $L_{\rho, 1}$ on the order of the fuel radius or less, we expect that $L_{\rho, 2}$ should lie in the range of 1-10 microns.

The time-dependence of $\sigma_{1}$ can be obtained from mass conservation. Using Eq. (3) we have: $\sigma_{1}+3=4 \pi \bar{\rho}_{1,0} \cdot R^{3} / M_{1}$ where $M_{1}$ is the conserved fuel mass. If $d \ln \left(3+\sigma_{1}\right) / d t<<3 d \ln R / d t$ is satisfied, then $\bar{\rho}_{1,0} \propto 1 / R^{3}$ and $\sigma_{1} \approx$ const. For example, this condition holds exactly when $L_{\rho, 1} \approx R$. For $\sigma_{2} \equiv R / L_{\rho, 2}$ we find from Eq. (8c) with $Z_{2}>>1$ that $L_{\rho, 2} \approx L_{\rho, 1} \cdot Z_{2}^{-9 / 2} \cdot\left(\bar{\rho}_{1,0} / \bar{\rho}_{2,0}\right)^{7 / 2}$. For radiation temperatures up to several hundred $\mathrm{eV}$, we apply the following scaling for gold [2]: $Z_{2} \propto T_{R}^{0.45}$. The radiation flux in the fuel scales as $R^{-3}$, giving $T_{R} \propto R^{-3 / 4}$. Using that $\bar{\rho}_{1,0} / \bar{\rho}_{2,0} \propto 1 / R$ and $L_{\rho, 1} \approx R$ from above, we finally obtain $L_{\rho, 2} \propto 1 / R$ and $\sigma_{2} \propto R^{2}$. Figure $2 \mathrm{~b}$ shows the time history of $\sigma_{1}$ and $\sigma_{2}$ between deceleration onset and the instant of minimum fuel volume. Indeed, $\sigma_{1}$ is found to remain nearly constant throughout the deceleration stage except for brief instances when a shock transits the interface. We find also that $\sigma_{2}$ varies strongly with fuel radius, in qualitative agreement with the above scaling.

Another example of an ICF-relevant target is an indirectly-driven, germanium-doped, plastic $\left(\mathrm{CHGe}_{0.02}\right)$, Omega-scale, single-shell capsule containing $10 \mathrm{~atm}$ of deuterium fuel [19]. Figure $2 \mathrm{c}$ shows the simulated density-gradient scalelengths after deceleration onset. Throughout the deceleration stage we notice that $L_{\rho, 1} / L_{\rho, 2} \approx 2-3$, in fair agreement with Eq. (8c) for $Z_{2} \approx 3.5, Z_{1} \approx 1$.

In summary, Eqs. $(5,8 \mathrm{c})$ represent two candidate sources of contiguous density-gradient scalelengths for input to a Plesset-type fluid model. In the following we adapt such a fluid formulation to accommodate contiguous density-gradient scalelengths on a classical interface.

\section{b) Unperturbed potential flow}


To find the unperturbed flow within the two regions of interest we introduce a velocity potential $\Psi_{i, 0}$ that satisfies [15]: $\vec{v}_{i, 0}=-\vec{\nabla} \Psi_{i, 0}$, where the "0" subscript denotes the unperturbed flow. We insert the trial solution for the fluid density from Eq. (3) in the mass continuity equation $\partial_{t} \rho_{i, 0}+\vec{\nabla} \cdot\left(\rho_{i, 0} \vec{v}_{i, 0}\right)=0$ to obtain:

$$
\frac{d \ln \bar{\rho}_{i, 0}}{d t}-\sigma_{i} \frac{d \ln R}{d t}+\frac{d \sigma_{i}}{d t} \ln (r / R)-\frac{\sigma_{i}}{r} \partial_{r} \Psi_{i, 0}-\nabla^{2} \Psi_{i, 0}=0 .
$$

The solution to Eq. (9) in region 1 satisfies regularity at the origin and is given by:

$$
\Psi_{1,0}=\frac{\bar{F}_{1} r^{2}}{6}+\frac{d \sigma_{1}}{d t} \cdot \frac{r^{2}}{2\left(\sigma_{1}+3\right)}\left[\ln \left(\frac{r}{R}\right)-\frac{\sigma_{1}+5}{2\left(\sigma_{1}+3\right)}\right],
$$

where

$$
\bar{F}_{1} \equiv \frac{\frac{d \ln \bar{\rho}_{1,0}}{d t}-\sigma_{1} \frac{d \ln R}{d t}}{1+\frac{\sigma_{1}}{3}}=F_{1}+\frac{d \sigma_{1}}{d t} \cdot \frac{3}{\left(\sigma_{1}+3\right)^{2}}
$$

$F_{1} \equiv d \ln \bar{\rho}_{1,0} / d t=-3 d \ln R / d t$, and the final identity in Eq. (10b) follows from applying mass conservation to the enclosed fluid. Note that the flow is self-similar $\left(\Psi_{1} \propto r^{2}\right)$ as a result. For region 2 we similarly obtain:

$$
\begin{aligned}
\Psi_{2,0}= & \frac{\bar{F}_{2} r^{2}}{6}+\left(\frac{R}{r}\right)^{1+\sigma_{2}} \cdot \frac{R^{2}}{1+\sigma_{2}} \cdot\left[\frac{\left(\bar{F}_{2}-\bar{F}_{1}\right)}{3}+\frac{d \sigma_{1}}{d t} \cdot \frac{1}{\left(3+\sigma_{1}\right)^{2}}-\frac{d \sigma_{2}}{d t} \cdot \frac{1}{\left(3+\sigma_{2}\right)^{2}}\right]+ \\
& \frac{d \sigma_{2}}{d t} \cdot \frac{r^{2}}{2\left(\sigma_{2}+3\right)} \cdot\left[\ln \left(\frac{r}{R}\right)-\frac{\left(\sigma_{2}+5\right)}{2\left(\sigma_{2}+3\right)}\right],
\end{aligned}
$$

where 


$$
\bar{F}_{2} \equiv \frac{\frac{d \ln \bar{\rho}_{2,0}}{d t}-\sigma_{2} \frac{d \ln R}{d t}}{1+\frac{\sigma_{2}}{3}}
$$

In deriving Eq. (11a) we have imposed the condition that the fluid velocity normal to the unperturbed interface be continuous. The solutions embodied in Eqs. (10-11) constitute a potential-density pair $\left\{\Psi_{i, 0}, \rho_{i, 0}\right\}$ that determines the unperturbed flow on either side of the interface. The occurrence of density gradients in each region is characterized by the parameter $\sigma_{i}$, which in turn is related to the value of the density gradient at the interface, cf., Eq. (2). For the case of homogeneous fluids $\left(\sigma_{i}=0\right)$, Eqs. (10-11) recover former results [12]. We note also that the flow in region 2 is generally not self-similar, in contrast to region 1 .

\section{c) Perturbed potential flow}

Out main interest is the study of perturbation growth where the associated wavelength is on the order of a density-gradient scalelength or larger. In this regime the Rayleigh-

Taylor growth rate scales as $\sqrt{g / L_{\rho}}$. Using Eq. (5) for the density-gradient scalelength as an example, we obtain that an $e$-folding time is on the order of an acoustic transit time across the perturbation wavelength. Thus, compressible perturbation growth needs to be considered in this regime of interest.

We now perturb the density and velocity potential in each region as follows: $\rho_{i}=\rho_{i, 0}+\delta \rho_{i}, \Psi_{i}=\Psi_{0, i}+\delta \Psi_{i}$, giving for the linearized continuity equation:

$$
\frac{d}{d t} \delta \rho_{i}-\delta \rho_{i} \nabla^{2} \Psi_{i, 0}=\rho_{i, 0} \nabla^{2} \delta \Psi_{i}+\vec{\nabla} \delta \Psi_{i} \cdot \vec{\nabla} \rho_{i, 0}
$$

In both Bell's and Plesset's original analysis for homogeneous incompressible fluids [15], the perturbed density was neglected $\left(\delta \rho_{i}=0\right)$ and the perturbed continuity equation (12) reduced to Laplace's equation: $\nabla^{2} \delta \Psi_{i}=0$. In Amendt et al. [12], the bulk flow was 
assumed compressible $\left(\nabla^{2} \Psi_{i, 0} \neq 0\right)$, but the perturbations were also taken as incompressible $\left(\nabla^{2} \delta \Psi_{i}=0\right)$. In this present work the fluid perturbations are assumed to be generally compressible in the presence of background density gradients $\left(\sigma_{i} \neq 0\right)$. To clarify our assumptions we introduce an expansion parameter $\varepsilon_{i} \equiv \delta \rho_{i} / \rho_{i, 0}<<1$ and expand the perturbed potential: $\delta \Psi_{i}=\delta \Psi_{i}^{(0)} \varepsilon_{i}^{0}+\delta \Psi_{i}^{(1)} \varepsilon_{i}^{1}+\ldots$. In order $\varepsilon_{i}^{0}$ and $\varepsilon_{i}^{1}$ we obtain from Eq. (12):

$$
\begin{gathered}
\nabla^{2} \delta \Psi_{i}^{(0)}+\vec{\nabla} \delta \Psi_{i}^{(0)} \cdot \vec{\nabla} \ln \rho_{i, 0}=0, \quad(13 a) \\
\frac{d}{d t} \delta \rho_{i}-\delta \rho_{i} \nabla^{2} \Psi_{i, 0}=\vec{\nabla} \delta \Psi_{i}^{(1)} \cdot \vec{\nabla} \rho_{i, 0}+\rho_{i, 0} \nabla^{2} \delta \Psi_{i}^{(1)}
\end{gathered}
$$

Thus, if

$$
\nabla^{2} \delta \Psi_{i}+\vec{\nabla} \delta \Psi_{i} \cdot \vec{\nabla} \ln \rho_{i, 0}=0
$$

holds to order $\varepsilon_{i}^{1}$ as well, then we obtain in addition the closure relation:

$$
\frac{d}{d t} \delta \rho_{i}=\delta \rho_{i} \nabla^{2} \Psi_{i, 0}
$$

Equation (14a) relates the compressibility of the perturbation to the compressibility of the background flow, i.e., $\sigma_{i} \neq 0$, and serves as the foundation for the following analysis of perturbation growth.

The solution of Eq. (14a) is obtained by first substituting the following trial solution: $\delta \Psi_{i}(r, \theta) \equiv \delta \psi_{i}(r) \cdot \Theta_{i}(\theta)$, giving

$$
r \cdot\left(2+\sigma_{i}\right) \frac{\partial_{r} \delta \psi_{i}}{\delta \psi_{i}}+r^{2} \cdot \frac{\partial_{r r}^{2} \delta \psi_{i}}{\delta \psi_{i}}=-\frac{\partial_{\theta}\left(\sin \theta \cdot \partial_{\theta} \Theta_{i}\right)}{\Theta_{i} \sin \theta} \equiv \ell(\ell+1)
$$


The solution to Eq. (15) then readily follows:

$$
\delta \Psi_{i}=P_{\ell}(\cos \theta) \cdot r^{n_{i \pm}}, \quad(16 a)
$$

where $P_{\ell}$ is the Legendre polynomial of order $\ell$ and

$$
n_{i \pm}=\frac{-\left(1+\sigma_{i}\right)}{2} \pm \frac{1}{2} \cdot \sqrt{(2 \ell+1)^{2}+\sigma_{i}\left(2+\sigma_{i}\right)} .
$$

Two limiting cases are of interest. For $\left|\sigma_{i}\left(2+\sigma_{i}\right)\right|<<(2 \ell+1)^{2}$, we obtain:

$$
\begin{gathered}
n_{i+} \cong \ell-\sigma_{i} / 2+\frac{\sigma_{i}\left(\sigma_{i}+2\right)}{2(2 \ell+1)^{2}}, \\
n_{i-} \cong-\ell-1-\sigma_{i} / 2-\frac{\sigma_{i}\left(\sigma_{i}+2\right)}{2(2 \ell+1)^{2}},
\end{gathered}
$$

and for $\left|\sigma_{i}\left(2+\sigma_{i}\right)\right|>>(2 \ell+1)^{2}$ we have:

$$
\begin{gathered}
n_{i+} \cong \frac{(2 \ell+1)^{2}}{4 \sigma_{i}}, \\
n_{i-} \cong-\sigma_{i}-\frac{(2 \ell+1)^{2}}{4 \sigma_{i}} .
\end{gathered}
$$

We are mainly interested in surface-type perturbation solutions, e.g., RT, that remain localized near the interface. This requirement leads to the following conditions for the eigenfunctions of Eq. (15): $n_{1} \equiv \max \left(n_{1 \pm}\right)>0$ in region 1 and $n_{2} \equiv \min \left(n_{2 \pm}\right)<0$ in region 2. A further set of conditions on our solution consists of (1) imposing continuity of the normal component of the fluid velocity across the interface and (2) ensuring that the transverse fluid motion matches the interface motion. These two conditions take the following form:

$$
-\left(\frac{\partial \Psi_{1}}{\partial r}\right)_{r=r_{S}}=-\left(\frac{\partial \Psi_{2}}{\partial r}\right)_{r=r_{S}}=\frac{d R}{d t}+\frac{d a_{\ell}}{d t} \cdot P_{\ell}(\cos \theta),
$$


where the radius of the perturbed interface $r_{s}$ satisfies: $r_{s}=R+a_{\ell} P_{\ell}(\cos \theta)$. Applying Eqs. (19) to Eq. (16a) for the perturbed solutions and including for completeness the unperturbed solutions from Eqs. (10a, 11a), we obtain for the full potential solutions in regions 1, 2:

$$
\Psi_{1}=\frac{\bar{F}_{1} r^{2}}{6}+\frac{d \sigma_{1}}{d t} \cdot \frac{r^{2}}{2\left(\sigma_{1}+3\right)}\left[\ln \left(\frac{r}{R}\right)-\frac{\sigma_{1}+5}{2\left(\sigma_{1}+3\right)}\right]-\frac{R^{1-n_{1}}}{n_{1}} \cdot\left(\frac{d a_{\ell}}{d t}+\frac{\bar{F}_{1}}{3} a_{\ell}\right) \cdot r^{n_{1}} P_{\ell}(\cos \theta)
$$

and

$$
\begin{aligned}
\Psi_{2}= & \frac{\bar{F}_{2} r^{2}}{6}+\left(\frac{R}{r}\right)^{1+\sigma_{2}} \cdot \frac{R^{2}}{1+\sigma_{2}} \cdot\left[\frac{\left(\bar{F}_{2}-\bar{F}_{1}\right)}{3}+\frac{d \sigma_{1}}{d t} \cdot \frac{1}{\left(3+\sigma_{1}\right)^{2}}-\frac{d \sigma_{2}}{d t} \cdot \frac{1}{\left(3+\sigma_{2}\right)^{2}}\right]+ \\
& \frac{d \sigma_{2}}{d t} \cdot \frac{r^{2}}{2\left(3+\sigma_{2}\right)} \cdot\left[\ln \left(\frac{r}{R}\right)-\frac{\left(\sigma_{2}+5\right)}{2\left(3+\sigma_{2}\right)}\right]- \\
& \left(\frac{r}{R}\right)^{n_{2}} \cdot \frac{R}{n_{2}} \cdot\left[\frac{d a_{\ell}}{d t}+\frac{\bar{F}_{2}}{3} a_{\ell}+\frac{\left(2+\sigma_{2}\right)}{3}\left(\bar{F}_{2}-\bar{F}_{1}\right) a_{\ell}\right] P_{\ell}(\cos \theta) .
\end{aligned}
$$

At this point we address to what extent a low-order mode is potentially affected by a spatially varying density-gradient scalelength. We focus the discussion on the case of positive $\sigma_{i}$ which generally holds in the case of ICF implosions where thermal conduction from the hot fuel to the cooler pusher establishes a positive density gradient [See Fig. 2a]. From the discussion below Eq. (5) we have that $L_{\rho, i}$ varies linearly with radius away from the interface independent of the nature of the density gradient. Thus, a lowest-order mode will sample an appreciable variation in density between the interface position and the origin, cf., Eq. (18a). However, in a density-weighted sense the variation of $L_{\rho, 1}$ from its interface value is generally small: $\left\langle L_{\rho, 1}\right\rangle_{\rho}=L_{\rho, 1}(R) \cdot\left(\sigma_{1}+4\right) /\left(\sigma_{1}+5\right)$, leading only to a $20 \%$ difference at most. Therefore, we expect only a modest effect from a power-law density variation [Eq. (3)] on the perturbation profile inside the interface. Outside the interface in region 2, we find from Eq. (18b) that the effective radial extent of 
the perturbation is greatly reduced, falling off as $r^{-\sigma_{2}}$ where $\sigma_{2} \gg 1$ typically holds. Consequently, the perturbation profile effectively samples a distance into the outer density profile at most a small fraction of $L_{\rho, 2}(R)$. We stress that the linearized solutions represented by Eqs. (16a-b) implicitly include the effect of a spatially varying densitygradient scalelength and hold exactly over any distance from the interface, modulo the usual limits of a linear analysis.

\section{d) Bernoulli's equation}

The evolution of the perturbation amplitude $a_{\ell}$ in Eqs. $(20 \mathrm{a}, \mathrm{b})$ is found by applying momentum balance and then imposing pressure balance across the interface with use of Bernoulli's equation. Assuming that the two fluids are polytropes $\left(P_{i} \rho_{i}^{-\gamma_{i}}=C_{i}\right)$ we find that Bernoulli's equation takes the form:

$$
\left[\frac{\partial \Psi_{i}}{\partial t}-\frac{\left|\bar{\nabla} \Psi_{i}\right|^{2}}{2}\right]_{r=r_{S}}-\frac{\gamma_{i}}{\gamma_{i}-1} \cdot\left[\frac{P_{i}\left(r_{S}\right)}{\rho_{i}\left(r_{S}\right)}-\frac{P_{i}\left(r_{i}\right)}{\rho_{i}\left(r_{i}\right)}\right]=0
$$

where $P_{1}\left(r_{1}\right) \equiv P_{1}(0), P_{2}\left(r_{2}\right) \equiv P_{2}\left(r>r_{s}\right)$ for the interior and exterior pressures, and $\gamma_{i}>1$. Because of the adopted form of the density profile [Eq. (3)], necessarily $P_{1}(0)=0$. In region 2 , the density is monotone increasing or decreasing depending on the sign of $\sigma_{2}$. We need only consider $r_{2}$ several wavelengths at most removed from the interface in order to adequately capture the behavior of a given surface perturbation; indeed, as we show below, the external pressure $P_{2}\left(r_{2}\right)$ cancels out exactly in a linearized analysis. The unperturbed motion of the interface naturally depends on the specified pressure difference between $r_{2}$ and the origin.

The underlying assumption in our above analysis is that the fluid is ideal, i.e., viscosity and thermal conduction may be neglected. For a compressible, adiabatic, ideal fluid we identify $\gamma_{i}$ above with the usual ratio of specific heats $c_{P} / c_{V}$. The assumption of a 
polytropic fluid is also convenient as an analytic device for recovering incompressible fluid behavior as $\gamma_{i} \rightarrow \infty$, allowing for direct comparison with previous work [15].

We next derive the linearized form of Eq. (21) on the perturbed interface. Using the unperturbed solution for Eq. (21) and the total perturbed density variation on the displaced boundary [16]

$$
\delta \rho_{i}=\sigma_{i} \bar{\rho}_{i, 0} \cdot\left(\frac{a_{\ell}}{R}\right) \cdot P_{\ell}(\cos \theta)+\frac{\delta P_{i}}{C_{s, i}^{2}},
$$

we straightforwardly obtain:

$$
\begin{array}{r}
\delta P_{1}=\rho_{1,0}^{\prime} \cdot\left(\delta D_{1}+\sigma_{1} \cdot D_{1} \cdot\left(\frac{a_{\ell}}{R}\right) P_{\ell}(\cos \theta)\right), \quad(23 a) \\
\delta P_{2}=\rho_{2,0}^{\prime} \cdot \delta D_{2}+\sigma_{2} \cdot \rho_{1,0}^{\prime} \cdot D_{1} \cdot\left(\frac{a_{\ell}}{R}\right) \cdot\left(\frac{1-\frac{D_{1}}{C_{s, 1}^{2}} \cdot \frac{\left(\gamma_{1}-1\right)}{\gamma_{1}}}{1-\frac{D_{1}}{C_{s, 1}^{2}} \cdot \frac{\left(\gamma_{1}-1\right)}{\gamma_{2}}}\right) P_{\ell}(\cos \theta),
\end{array}
$$

where

$$
\begin{array}{r}
D_{1} \equiv\left[\frac{\partial \Psi_{1,0}}{\partial t}-\bar{\nabla} \Psi_{1,0} \cdot \bar{\nabla} \Psi_{1,0}\right]_{r=R}=-\frac{R g}{2}-\frac{d}{d t}\left[\frac{d \sigma_{1}}{d t} \cdot \frac{R^{2}}{4\left(\sigma_{1}+3\right)}\right] \\
\delta D_{i} \equiv\left[\frac{\partial \delta \Psi_{i}}{\partial t}-\bar{\nabla} \delta \Psi_{i} \cdot \bar{\nabla} \Psi_{i, 0}\right]_{r=r_{S}}, \quad(24 b)
\end{array}
$$

and

$$
\rho_{1,0}^{\prime} \equiv \bar{\rho}_{1,0} \cdot \frac{\left(\gamma_{1}-1\right)}{\gamma_{1}} \cdot \frac{1}{\left(1-\frac{\left(\gamma_{1}-1\right)}{\gamma_{1}} \cdot \frac{D_{1}}{C_{s, 1}^{2}}\right)},
$$




$$
\rho_{2,0}^{\prime} \equiv \bar{\rho}_{2,0} \cdot \frac{\left(\gamma_{2}-1\right)}{\gamma_{2}} \cdot \frac{1}{\left(1-\frac{\left(\gamma_{1}-1\right)}{\gamma_{2}} \cdot \frac{D_{1}}{C_{s, 1}^{2}}\right)}
$$

for the renormalized densities. In obtaining Eqs. (23b, 25b) we have made use of the identity: $\gamma_{2} \bar{\rho}_{1,0} C_{s, 1}^{2}=\gamma_{1} \bar{\rho}_{2,0} C_{s, 2}^{2}$, which follows from applying pressure balance across the unperturbed interface. We point out that the external pressure $P_{2}\left(r_{2}\right)$ conveniently cancels out in the above linearized analysis. The general dispersion relation follows from imposing pressure balance across the perturbed interface in Eqs. (23a-b), i.e., $\delta P_{1}=\delta P_{2}$. We note the occurrence of two resonances in Eqs. (25a-b) for $D_{1}>0$, e.g., when $g<0$ and $d \sigma_{1} / d t \approx 0$. For the illustrative example of a uniformly accelerating shell [See Eq. (6)] we can write for the location of the pair of resonances:

$$
\sigma_{i}=-\frac{2 \gamma_{i}}{\gamma_{1}-1}
$$

Thus, the existence of a resonance between the interface radius and density-gradient scalelength requires suitably large density gradients such that $\sigma_{i}<-2$ for $1<\gamma_{i}<\infty$. We note that the potential existence of this pair of resonances is entirely derived from Bernoulli's Equation with no recourse to the continuity equation or closure hypothesis [Eqs. (14a-b)].

By virtue of the sign of $D_{1}$ in general, potential resonance effects in a spherical implosion are manifested only during acceleration $g<0(d R / d t<0)$ of the fuel/pusher interface. This condition precludes the occurrence of RT instability, leaving only Richtmyer-Meshkov (RM) instability [20] as a possible venue for resonance phenomena. However, the growth rate for this instability is proportional to the Atwood number, which depends on the density ratio $\rho_{2}^{\prime} / \rho_{1}^{\prime}$. Using Eqs. (25a-b) to evaluate this ratio, the resonant denominators are seen to cancel out only if $\gamma_{1}=\gamma_{2}$. Thus, differing values of the 
adiabatic index across an accelerating fuel/pusher interface may potentially lead to enhanced RM growth according to a linearized analysis of the Bernoulli equation.

Applying Eq. (21) to both sides of the interface leads to an interesting issue when we enforce pressure equilibrium. How will the density profiles adjust to the perturbed interface? In other words, will the density stratify along the perturbed interface or the unperturbed interface [See Fig. 3]? This distinction will lead to two distinct physical regimes of interest as follows. The time-scale for the perturbed density to change significantly is defined as [21]:

$$
\tau_{\rho, i} \equiv\left|\left(d \ln \delta \rho_{i} / d t\right)^{-1}\right|_{r=R}=\left|\nabla^{2} \Psi_{i, 0}\right|_{r=R}^{-1},
$$

where the last identity follows directly from Eq. (14b). Using Eqs. (10a, 11a) we can readily evaluate Eq. (26):

$$
\tau_{\rho, i}=\left|F_{i}\right|^{-1}
$$

where $F_{i} \equiv d \ln \bar{\rho}_{i, 0} / d t$. With $F_{1}=-3 d \ln R / d t$ from conservation of fuel mass and $F_{2} \approx-2 d \ln R / d t$ for a compressible shell [12], $\min \left(\tau_{\rho, i}\right) \cdot d \ln R / d t \approx 1 / 3$. For classical RT growth we define the threshold for an "incompressible" perturbation response when $\min \left(\tau_{\rho, i}\right) \cdot \gamma_{R T, c}>1$ or:

$$
\ell>\ell_{c} \equiv 9 \cdot\left[\frac{(d R / d t)^{2}}{A t \cdot g \cdot R}\right] .
$$

For typical ICF conditions with $g \approx 10^{3} \mu \mathrm{m} / \mathrm{ns}^{2}, d R / d t \equiv v_{0} \approx 10^{2} \mu \mathrm{m} / \mathrm{ns}$, and $R \approx 10$ $100 \mu \mathrm{m}$, we find a threshold mode number from Eq. (28) $\ell \approx 1-10$. Thus, for mode numbers on the order of $\approx 10$ or greater, the RT perturbation response is essentially 
incompressible for most ICF applications and leads to stratification of the density parallel to the perturbed interface. In the following we distinguish between these two regimes.

\section{e) Incompressible regime}

We now evaluate Eq. (21) with constant density on the perturbed interface, i.e., $\rho_{i}\left(r_{S}\right) \approx \rho_{i}(R)$. For the unperturbed motion of the interface we obtain the fairly complicated relation:

$$
\begin{aligned}
\rho_{1,0}^{\prime}\left[\frac{1}{2}\left(\frac{F_{1} R}{3}\right)^{2}-\frac{d F_{1}}{d t} \frac{R^{2}}{6}+\frac{d}{d t}\left(\frac{R^{2}}{4\left(\sigma_{1}+3\right)} \cdot \frac{d \sigma_{1}}{d t}\right)\right]= \\
\quad \rho_{2,0}^{\prime}\left[\frac{1}{2}\left(\frac{F_{1} R}{3}\right)^{2}-\frac{d \bar{F}_{2}}{d t} \frac{R^{2}}{6}+\frac{d R}{d t} \cdot \frac{\left(3+\sigma_{2}\right)}{\left(1+\sigma_{2}\right)} \cdot\left(\frac{\overline{F_{2}}-\bar{F}_{1}}{3}+\frac{d \sigma_{1}}{d t} \cdot \frac{1}{\left(3+\sigma_{1}\right)^{2}}-\frac{d \sigma_{2}}{d t} \cdot \frac{1}{\left(3+\sigma_{2}\right)^{2}}\right) \cdot R+\right. \\
\frac{R^{2}}{1+\sigma_{2}} \cdot \frac{d}{d t}\left(\frac{\left(\bar{F}_{2}-\bar{F}_{1}\right)}{3}+\frac{d \sigma_{1}}{d t} \cdot \frac{1}{\left(3+\sigma_{1}\right)^{2}}-\frac{d \sigma_{2}}{d t} \cdot \frac{1}{\left(3+\sigma_{2}\right)^{2}}\right)-\frac{R^{2}\left(5+\sigma_{2}\right)}{4\left(3+\sigma_{2}\right)} \cdot \frac{d}{d t}\left(\frac{d \sigma_{2}}{d t} \cdot \frac{1}{\left(3+\sigma_{2}\right)}\right)- \\
\left.\frac{R}{2\left(3+\sigma_{2}\right)} \cdot \frac{d R}{d t} \cdot \frac{d \sigma_{2}}{d t}+\frac{R^{2}}{2\left(3+\sigma_{2}\right)^{3}} \cdot\left(\frac{d \sigma_{2}}{d t}\right)^{2}+\frac{P\left(r_{2}\right)}{\rho_{2}\left(r_{2}\right)}\right] .
\end{aligned}
$$

We will not make further use of Eq. (29) here; rather, we concentrate on the companion perturbation equation that we now derive. Upon enforcing pressure balance on the perturbed interface with use of Eq. (21), collecting terms proportional to $a_{\ell}$, and then carrying through some straightforward algebra, we find

$$
\begin{aligned}
\frac{d^{2} a_{\ell}}{d t^{2}}+\frac{d a_{\ell}}{d t} \cdot\left[\beta_{12} \cdot\left(\frac{d \ln n_{1}}{d t}-\frac{d \ln \left(3+\sigma_{1}\right)}{d t}\right)-\beta_{21} \cdot\left(\frac{d \ln n_{2}}{d t}+(\alpha-3) \cdot \frac{d \ln R}{d t}\right)\right]- \\
a_{\ell} \cdot\left[\frac{g}{R} \cdot\left((\ell-1) A_{\ell}-\beta_{21}(2-\alpha)-\frac{3 \beta_{22}}{3+\sigma_{1}}\right)+\beta_{21} \cdot \frac{d \ln R}{d t} \cdot \frac{d \alpha}{d t}+\beta_{12} \cdot G_{1}-\beta_{21} \cdot G_{2}\right]=0
\end{aligned}
$$

where 


$$
\begin{array}{r}
\beta_{i j}=\frac{\rho_{i, 0}^{\prime} n_{j}}{\rho_{2,0}^{\prime} n_{1}-\rho_{1,0}^{\prime} n_{2}}, \\
\alpha \equiv-\frac{d \ln \bar{\rho}_{2,0}}{d \ln R}
\end{array}
$$

as in Ref. 12,

$$
A_{\ell} \equiv \frac{\left(\beta_{12}-\beta_{22}\right)}{\ell-1} \cdot\left(n_{1}-\frac{3}{3+\sigma_{1}}\right)
$$

is a generalized modal Atwood number, and

$$
G_{1} \equiv n_{1} \cdot \frac{d \ln \sigma_{1}}{d t} \cdot \frac{d}{d t}\left(\frac{\sigma_{1}}{n_{1}\left(3+\sigma_{1}\right)}\right)+\frac{d \ln R}{d t} \cdot \frac{d \ln n_{1}}{d t}-\frac{\sigma_{1}}{\left(3+\sigma_{1}\right) R} \cdot \frac{d}{d t}\left(R \frac{d \ln L_{\rho, 1}}{d t}\right)
$$

and

$$
G_{2} \equiv \frac{d \sigma_{2}}{d t} \cdot\left(\frac{\bar{F}_{2}-F_{1}}{3}\right)-\frac{d \ln n_{2}}{d t} \cdot \frac{\left(\bar{F}_{2}+\left(2+\sigma_{2}\right)\left(\bar{F}_{2}-F_{1}\right)\right)}{3}-\frac{d \ln R}{d t} \cdot \frac{d \sigma_{2}}{d t} \cdot \alpha \cdot(3-\alpha),
$$

include explicit acceleration-free, density-gradient terms that vanish as $\sigma_{i} \rightarrow 0$. Equation (30) is only modestly more complicated than former results in the absence of density gradients $\left(\sigma_{i}=0\right)$ [12]. We highlight some of the differences. The term in Eq. (30) proportional to $d a_{\ell} / d t$ is traditionally identified as a source of classical BP growth due to geometrical convergence (or divergence) effects. We see from Eq. (30) that this term is significantly changed from the homogeneous result with the addition of several terms ( $\propto d \sigma_{i} / d t$ ) having a mild mode number dependence. These terms go generally in the direction of increasing BP growth - more so for the lowest mode numbers. Further geometric effects are found in the expression proportional to $a_{\ell} \beta_{i j}$ in Eq. (30). These latter terms are identified as intrinsically geometric due to the fact that they vanish in the planar limit [22]: $\ell, R \rightarrow \infty \ni \ell / R \equiv k<\infty$. The lone term that does survive in the planar 
limit is proportional to $g \cdot A_{\ell}$ and is identified as the source term for RT and RM growth. The definition of modal Atwood number [See Eq. (33)] was chosen to isolate and maintain the intrinsic $\rho_{2}-\rho_{1}$ dependence even at low mode numbers. Still, this term retains some geometric character, particularly at the lowest mode numbers. Only in the limit of large $\ell$ does this term recover a pure Atwood number dependence. It is worthwhile mentioning that recent work by Epstein [23], using a judicious renormalization of the perturbation amplitudes, succeeds in distinguishing pure RT behavior from BP growth in the case of like-compressible, homogeneous fluids.

Equation (30) simplifies greatly when $d \sigma_{i} / d t=0$, giving

$$
\begin{aligned}
0= & \frac{d^{2} a_{\ell}}{d t^{2}}+\frac{d a_{\ell}}{d t} \cdot \frac{d \ln R}{d t} \cdot(3-\alpha) \beta_{21}+ \\
& \frac{a_{\ell}}{R\left(\rho_{2,0}^{\prime} n_{1}-\rho_{1,0}^{\prime} n_{2}\right)} \cdot\left\{g\left[\left(\rho_{2,0}^{\prime}-\rho_{1,0}^{\prime}\right) n_{1} n_{2}+\rho_{2,0}^{\prime}(2-\alpha) n_{1}+\rho_{1,0}^{\prime} n_{2}\right]-\frac{d \ln R}{d t} \cdot \frac{d \alpha}{d t}\right\}
\end{aligned}
$$

We note that Eq. (36) is formally identical to the $\sigma_{i}=0$ case [12], apart from a remaining dependence of $n_{i}$ on $\sigma_{i}$ through Eq. (16b).

\section{f) Compressible regime}

We now derive the compressible analog of Eq. (30). Returning to Eq. (3), the stratified density at the modulated interface follows the prescription:

$$
\rho_{i}\left(r_{S}\right) \cong \bar{\rho}_{i, 0}(t) \cdot\left[1+\sigma_{i} \frac{a_{\ell}}{R} P_{\ell}(\cos \theta)\right]
$$

Using Eq. (37) in Eq. (21) reproduces Eq. (30) except for an addition to the term responsible for RT growth:

$$
\frac{g A_{\ell}}{R} \rightarrow \frac{g A_{\ell}}{R}+\frac{\beta_{12}}{R^{2}} \cdot\left(\sigma_{2}-\sigma_{1}\right) \cdot n_{1} D_{1} .
$$


For typical ICF conditions we can establish that the effect of compressible perturbations helps to reduce RT growth rates after deceleration onset. In general, $\beta_{12}<0, D_{1}<0, \sigma_{2}>\sigma_{1}>0$, and we quite reasonably assume that $\left|\sigma_{i}\left(2+\sigma_{i}\right)\right|>>(2 \ell+1)^{2}$ holds in the compressible regime, cf., Eq. (28). Using Eqs. (17a$\mathrm{b}, 24 \mathrm{a}, 31,38)$ along with the assumption $d \sigma_{1} / d t \approx 0$, we obtain the condition for suppressing (compressible) RT instability $\left(1 \leq \ell<\ell_{c}\right)$ :

$$
\sigma_{2}>\sigma_{1}+2\left(\frac{\rho_{2,0}^{\prime}}{\rho_{1,0}^{\prime}}-1\right)+\frac{8 \sigma_{1}}{3\left(3+\sigma_{1}\right)}
$$

Finally, our heuristic argument for delineating the compressible and incompressible regimes can be put on firmer footing by solving Eq. (22) for $\delta \rho_{i}$. For the simpler example of $\delta \rho_{1}$, we obtain:

$$
\frac{\delta \rho_{1}}{\bar{\rho}_{0,1}}=\frac{\left[\sigma_{1} \cdot\left(\frac{a_{\ell}}{R}\right)-\frac{1}{C_{s, 1}^{2}} \cdot\left(\frac{\gamma_{1}-1}{\gamma_{1}}\right)\left(\frac{R}{n_{1}} \frac{d^{2} a_{\ell}}{d t^{2}}+a_{\ell} g \cdot\left(1-\frac{1}{n_{1}}\right)\right)\right] P_{\ell}(\cos \theta)}{1+\left(\frac{\gamma_{1}-1}{\gamma_{1}}\right) \frac{R g}{2 C_{s, 1}^{2}}},
$$

where $\sigma_{1} \approx$ const. by assumption. For $\gamma_{1} \rightarrow 1$ we recover the compressible regime with the stratified perturbed density varying along the displaced interface in agreement with Eq. (37). For the incompressible limit $\gamma_{1} \rightarrow \infty$, we use that $C_{s, 1}^{2} \equiv \gamma_{1} C_{i, 1}^{2}$, where $C_{i, 1}$ is the isothermal sound speed in region 1. For the usual example [See Eq. (6)], we obtain: $\delta \rho_{1} / \bar{\rho}_{1,0} \propto\left(\gamma_{1} n_{1}\right)^{-1} \rightarrow 0$ as $\gamma_{1} \rightarrow \infty$. Thus, the perturbed density is shown in the incompressible limit to be constant on the perturbed interface as expected. A further limit of interest is $R \rightarrow \infty: \delta \rho_{1} / \bar{\rho}_{0,1} \rightarrow-2\left(d^{2} a_{\ell} / d t^{2}\right) / n_{1} g$. Using that $d^{2} a_{\ell} / d t^{2} \approx a_{\ell} \ell g \cdot A t / R$ for classical RT growth with $\ell>>1$, we obtain $\delta \rho / \bar{\rho}_{0,1} \rightarrow-2 A t \cdot a_{\ell} / R \rightarrow 0$ as $R \rightarrow \infty$. 


\section{Results}

We analyze in some detail the influence of contiguous density gradients on BP growth and the RT instability. With the compressible regime being restricted to relatively low mode numbers $\ell<10$, we concentrate on the incompressible case [Sec. IIe] which enjoys wider applicability for typical ICF conditions. In any case, if $\sigma_{1} \cong \sigma_{2} \equiv \sigma$ the distinction between the two regimes is rendered moot, cf., Eq. (38).

\section{a) Planar limit}

Before delving into the spherical and density-gradient effects predicted by our model, it is worth exploring the planar limit to compare with previous relevant work. Applying the planar limit to Eq. (30) in the usual manner results in

$$
\frac{d^{2} a_{\ell}}{d t^{2}}-a_{\ell} \cdot k g \cdot A_{k}=0
$$

where

$$
A_{k}=\frac{\left(\rho_{1,0}^{\prime}-\rho_{2,0}^{\prime}\right)\left(1-\sqrt{1+4 k^{2} L_{\rho, 1}^{2}}\right)\left(1+\sqrt{1+4 k^{2} L_{\rho, 2}^{2}}\right)}{2 k\left[\rho_{1,0}^{\prime} L_{\rho, 1} \sqrt{1+4 k^{2} L_{\rho, 2}^{2}}+\rho_{2,0}^{\prime} L_{\rho, 2} \sqrt{1+4 k^{2} L_{\rho, 1}^{2}}+\rho_{1,0}^{\prime} L_{\rho, 1}-\rho_{2,0}^{\prime} L_{\rho, 2}\right]} .
$$

For $\gamma_{1}=\gamma_{2}$ and in the large wavenumber limit $\left(4 k^{2} L_{\rho, i}^{2}>>1\right)$ Eqs. (41-42) recover the classical Rayleigh-Taylor growth rate. We mention that for $\gamma_{1} \neq \gamma_{2}$, instability persists even for equal densities $\left(\rho_{1,0}=\rho_{2,0}\right)$ and is driven by a pure entropy change across the interface [24]. In the small wavenumber limit, we obtain with aid of Eqs. (24a, 25a-b):

$$
\gamma_{R T}=k C_{s, 1} \cdot \sqrt{\frac{\rho_{2,0}^{\prime}}{\rho_{1,0}^{\prime}}-1} \rightarrow k C_{s, 1} \cdot \sqrt{\frac{C_{s, 1}^{2}}{C_{s, 2}^{2}}-1}
$$


as $D_{1} \rightarrow \infty$. Equation (43) is very similar to the fully compressible $\left(\gamma_{i}=1\right)$, planar result of Bernstein and Book [16], despite being strictly valid only in the incompressible regime $\left(\tau_{\rho, i} \cdot \gamma_{R T, c}>1\right)$. We note that the threshold mode number for compressibility onset $\ell_{c}$ vanishes in the planar limit for classical RT growth [See Eq. (28)], but remains finite in the large wavelength limit of incompressible RT growth [Eq. (43)]:

$$
\ell_{c}=\frac{3\left|\frac{d R}{d t}\right|}{C_{s, 1} \sqrt{\frac{\rho_{2,0}^{\prime}}{\rho_{1,0}^{\prime}}-1}} .
$$

Accordingly, we apply $R, \sigma_{i} \rightarrow \infty$ with $\ell<\ell_{c}$ to define the planar limit of compressible RT growth [Eq. (38)]:

$$
\gamma_{R T} \rightarrow \frac{(2 \ell+1) C_{s, 1}}{2 R} \sqrt{\frac{g R}{2} \cdot\left(\frac{1}{C_{s, 1}^{2}}-\frac{1}{C_{s, 2}^{2}} \cdot \frac{\gamma_{2}}{\gamma_{1}}\right)}
$$

vanishing as $R^{-1 / 2}$. Thus, truly compressible RT growth survives only in an intrinsically spherical geometry.

It is important to point out some key differences between the planar limit of an intrinsically spherical analysis such as ours and a pure slab treatment [16]. First, the interior solution of our spherical problem was manifestly constructed to conserve mass and behave regularly at the origin $[12,25,26]$. Such a construction implies that the problem is no longer symmetric upon interchange of the region indices $(1 \leftrightarrow 2)$ - in contrast to a pure planar treatment [16]. Second, our choice for the unperturbed density profile [Eq. (3)] in a uniformly accelerating shell agrees with previous planar treatments only when $\gamma_{i}=1$, which is precisely where our perturbation analysis breaks down [See Eq. (21)]. Third, previous planar treatments considered only a static geometry with no 
unperturbed fluid flows. However, such flows serve to physically delineate the perturbed compressible and incompressible regimes in our analysis - which would otherwise not be possible [See Eq. (28)]. Fourth, the form of Bernoulli's Equation (21) appropriate for a polytrope requires that the flow be isentropic (or characterized by constant entropy in space), in contrast to an intrinsically Lagrangian treatment [16] where the entropy is taken to be invariant along the trajectory of any fluid element. If, as usually happens, the entropy is uniform throughout the fluid volume at an initial time, the fluid maintains everywhere the same value of entropy for all subsequent times [27]. This isentropic assumption serves as the distinguishing feature of an analysis based on Bernoulli's Equation [See also Ref. 28] compared with prior Lagrangian-based analyses that obtained self-similar solutions with homogeneous deformation [29].

\section{b) BP effects}

We return to Eqs. (30-35) and assess the effect of density gradients on classical BP geometric growth. Two contributions from density gradients arise in the expression responsible for classical BP growth, i.e., the terms proportional to $d a_{\ell} / d t$ in Eq. (30). First, the occurrence of $\beta_{i j}$ includes the effect of density gradients through the dependence of $n_{j}$ on $\sigma_{j}$ [See Eq. (16b)]. We point out that this overall factor on BP growth $\left(\propto \beta_{i j}\right)$ arises solely because of the physical requirement that the velocity potential remain regular at the origin, cf., Eq. (10a). Such a requirement of self-similar flow within the interior fluid (fuel) leads to a linearly increasing radial velocity profile within the fuel, in agreement with radiation-hydrodynamics simulations [12]. Second, several of the terms are proportional to $d \sigma_{i} / d t=\sigma_{i} \cdot d \ln \left(R / L_{\rho, i}\right) / d t$ and represent an additional source for density-gradient effects. Figure 4 shows the cumulative change in classic BP growth due to nonzero density gradients $\left(\sigma_{1}=\sigma_{2} \equiv \sigma\right)$ compared to the homogeneous case, where $B_{\ell}$ is defined as the overall coefficient of $d a_{\ell} / d t \cdot d \ln R / d t$ in Eq. (30) [See Eq. (47) below]. Here, we have momentarily neglected the time dependence of $L_{\rho}$ for simplicity and used $\alpha \approx 2$ for a compressible shell [12]. We find 
that the classical BP growth rate is enhanced by the addition of positive density gradients, particularly for the lowest modes.

Continuing with our heuristic description of modified BP growth, we can carry through an analytical estimate by integrating the first two terms of Eq. (30) and assuming $R(t)=R_{0} \cdot\left(t / t_{0}\right)^{\mu}$ over a time interval $t-t_{0}$ :

$$
a_{\ell}(t)=a_{\ell}\left(t_{0}\right)+\left.\frac{d a_{\ell}}{d t}\right|_{t_{0}} \cdot \frac{t_{0}}{\mu B_{\ell}-1} \cdot\left[1-\left(\frac{t_{0}}{t}\right)^{\mu B_{\ell}-1}\right]
$$

where

$$
B_{\ell}(\sigma)=\frac{1}{\rho_{2} n_{1}-\rho_{1} n_{2}} \cdot\left[\frac{\sigma(1+\sigma)\left(\rho_{1}+\rho_{2}\right)}{2\left(n_{1}-n_{2}\right)}-\frac{\sigma\left(\rho_{2}-\rho_{1}\right)}{2}+\rho_{2} n_{1}(3-\alpha)-\frac{\sigma \rho_{1} n_{2}}{3+\sigma}\right],
$$

$\gamma_{1}=\gamma_{2}$ and $d \ln \left(L_{\rho} / R\right) / d t<<1$ are taken, and the unperturbed subscript on the density $\rho_{i}$ is now dropped for simplicity. We note that in the limit $\mu B_{\ell} \rightarrow 1$, Eq. (46) leads to logarithmic BP growth [12].

This initial discussion of geometric or BP growth only includes what is traditionally understood to form the basis of a classical BP analysis, namely, the middle term in Eq. (30). However, the last term $\left(\propto a_{\ell}\right)$ in Eq. (30) may also significantly contribute strictly geometric effects as we now consider some further examples.

We return to Eq. (30) and consider the behavior of low-order modes under strong density gradients such that $(2 \ell+1) \rho_{2} / \rho_{1} \ll 4 \sigma_{1} \sigma_{2}$. In this limit, taking $\alpha=2$ and again assuming $d \ln \left(L_{\rho i} / R\right) / d t<<1$, i.e., $d \sigma_{i} / d t \approx \sigma_{i} \cdot d \ln R / d t$, we find in leading order: 


$$
\begin{aligned}
0= & \frac{d^{2} a_{\ell}}{d t^{2}}+2 \frac{d a_{\ell}}{d t} \cdot \frac{d \ln R}{d t}+ \\
& a_{\ell}\left[\frac{\rho_{2} n_{1}}{\rho_{1} n_{2}}\left(2 \sigma_{2}\left(\frac{d \ln R}{d t}\right)^{2}-\frac{g n_{2}}{R}\right)-\left(\frac{3}{3+\sigma_{1}} \cdot \frac{d \ln R}{d t}\right)^{2}+\frac{g}{R}\left(n_{1}-\frac{3}{3+\sigma_{1}}\right)\right] .
\end{aligned}
$$

Using Eqs. (18a-b) for $n_{i}$, Eq. (48) becomes:

$$
\begin{aligned}
0= & \frac{d^{2} a_{\ell}}{d t^{2}}+2 \frac{d a_{\ell}}{d t} \cdot \frac{d \ln R}{d t}+ \\
& a_{\ell}\left[\frac{g}{R}\left(\frac{(2 \ell+1)}{4 \sigma_{1}} \cdot\left(1-\frac{\rho_{2}}{\rho_{1}}\right)-\frac{3}{3+\sigma_{1}}\right)-\left(\frac{d \ln R}{d t}\right)^{2} \cdot \frac{(2 \ell+1)}{2 \sigma_{1}} \cdot \frac{\rho_{2}}{\rho_{1}}\right]
\end{aligned}
$$

We note that in the limit $\sigma_{1} \rightarrow \infty$, the solution to Eq. (49) reduces to Eq. (46) with $B_{\ell}=2$. For large but finite $\sigma_{1}$ the solution to Eq. (49) is facilitated by treating the term in square brackets as a perturbation proportional to the small parameter $\varepsilon_{\sigma} \equiv 1 / \sigma_{1}\left(t_{0}\right)<<1$. Upon expanding $a_{\ell}=a_{\ell}^{(0)} \varepsilon_{\sigma}^{0}+a_{\ell}^{(1)} \varepsilon_{\sigma}^{1}+\ldots$ we trivially recover Eq. (46) in order $\varepsilon_{\sigma}^{0}$ with $B_{\ell}=2$. To order $\varepsilon_{\sigma}^{1}$ we straightforwardly find for $a_{\ell}^{(1)}$ using $R(t)=R_{0} \cdot\left(t / t_{0}\right)^{\mu}:$

$$
a_{\ell}^{(1)}=\varepsilon_{\sigma}^{1}\left[a_{\ell}^{(0)}+\frac{d a_{\ell}^{(0)}\left(t_{0}\right)}{d t} \cdot \frac{t_{0} H}{(3 \mu-1)(2 \mu-1)} \cdot\left(\frac{t}{t_{0}}\right)^{1-3 \mu}+\left(\frac{t}{t_{0}}\right)^{-\mu} H \cdot \frac{\left(a_{\ell}^{(0)}\left(t_{0}\right)+\frac{t_{0}}{(2 \mu-1)} \cdot \frac{d a_{\ell}^{(0)}\left(t_{0}\right)}{d t}\right)}{1-\mu}\right] \text {, }
$$

where

$$
H \equiv(1-\mu) \cdot\left[\left(\frac{\rho_{2}}{\rho_{1}}-1\right) \cdot \frac{(2 \ell+1)}{4}+3\right]-\mu \frac{(2 \ell+1)}{2} \cdot \frac{\rho_{2}}{\rho_{1}} .
$$


Equations $(46,50,51)$ describe combined BP and RT growth (for constant $L_{\rho i}$ ) in the low mode regime such that $(2 \ell+1) \rho_{2} / \rho_{1} \ll<\sigma_{1} \sigma_{2}$ is satisfied. For large $\sigma_{1}$ the lowmode growth is dominated by BP effects [Eq. (46)] with RT growth entering only in $\operatorname{order} \varepsilon_{\sigma}^{1}$ [Eq. (51)].

We now consider the complementary case of $L_{\rho i} \propto R$ such that $\sigma_{i}=$ constant. Defining $\varepsilon_{21}=\rho_{2} n_{1} / \rho_{1} n_{2}=\left(\rho_{2} / \rho_{1}\right)(2 \ell+1) /\left(4 \sigma_{1} \sigma_{2}\right) \ll<1$ with the aid of Eqs. (18a-b), we rewrite Eq. (36) as follows:

$$
\begin{aligned}
0= & \frac{d^{2} a_{\ell}}{d t^{2}}+\varepsilon_{21} \cdot \frac{d a_{\ell}}{d t} \cdot \frac{d \ln R}{d t} \cdot(3-\alpha)- \\
& a_{\ell} \cdot\left\{\frac{g}{R}\left[1+\sigma_{2} \varepsilon_{21} \cdot\left(1-\frac{\rho_{1}}{\rho_{2}}\right)+\varepsilon_{21} \cdot(2-\alpha)\right]-\varepsilon_{21} \cdot \frac{d \ln R}{d t} \cdot \frac{d \alpha}{d t}\right\}
\end{aligned}
$$

To zeroth order in $\varepsilon_{21}$ we obtain

$$
0=\frac{d^{2} a_{\ell}}{d t^{2}}-a_{\ell} \cdot \frac{g}{R}\left[1+\sigma_{2} \varepsilon_{21}\left(1-\frac{\rho_{1}}{\rho_{2}}\right)\right]
$$

where the last term is presumed to be of order unity for sufficiently large $\sigma_{2} \approx\left(\rho_{2} / \rho_{1}\right)(2 \ell+1) / 4$. To isolate the purely geometric behavior we set $\rho_{1}=\rho_{2}$, thereby manifesting a new source of BP growth in the presence of large (contiguous) density gradients. Assuming $R(t)=R_{0} \cdot\left(t / t_{0}\right)^{\mu}$ as before, we find purely accelerationdriven geometric growth

$$
a_{\ell}(t)=a_{\ell}\left(t_{0}\right) \cdot\left(\frac{t}{t_{0}}\right)^{\eta}
$$


where $\eta=\mu, 1-\mu$. Geometric growth occurs for $\mu<0$ during the deceleration or stagnation phase of an implosion $(g>0)$, while an accelerating unstable interface requires $\mu>0$.

The general solution of Eq. (52) can be readily found for the case of nearly constant $\rho_{1} / \rho_{2}$ as follows:

$$
a_{\ell}(t) \propto t^{\eta_{ \pm}},
$$

where

$$
\eta_{ \pm}=\frac{1}{2}-\frac{\varepsilon_{21} \mu(3-\alpha)}{2} \pm \sqrt{\frac{1}{4}\left(1-\varepsilon_{21} \mu(3-\alpha)\right)^{2}+\mu(\mu-1)\left(1+\varepsilon_{21} \sigma_{1}\left(1-\frac{\rho_{1}}{\rho_{2}}\right)+\varepsilon_{21}(2-\alpha)\right)}
$$

Secular growth ensues for $\max \left(\eta_{ \pm}\right)>0$. Note that Eqs. (55a-b) provide a clear example of the commonly encountered coupling between geometric and RT growth for finite radius $R$.

\section{c) RT and RM growth}

The term proportional to $g \cdot A_{\ell}$ in Eq. (30) is identified as the source for RT growth. The explicit effect of contiguous density gradients on RT growth is entirely contained in the modal Atwood number as defined in Eq. (33). In Figs. (5a-b) we plot the ratio of the RT source term normalized to the homogeneous case $\sqrt{A_{\ell}(\sigma) / A_{\ell}(0)}$ as a convenient measure of the effect of contiguous density gradients. Compared with the homogeneous case, we find an enhancement in the RT growth rate over a particular range of mode numbers. This effective increase in modal Atwood number is attributed to the sampling of larger density changes by the perturbation over a spatial scale on the order of a wavelength. Larger wavelengths sample more of the density profile away from the 
interface which leads to an effectively larger density jump for $\sigma>0$ and increased growth. We can analytically estimate the location $\ell_{\max }$ of the maximally enhanced growth with use of Eq. (33), assuming $\sigma, \ell>>1$ and $\gamma_{1}=\gamma_{2}$ :

$$
\ell_{\max } \cong \frac{\sigma \sqrt{1-A t^{2}}}{2 A t} .
$$

Using Eqs. $(33,56 a)$ we find for the enhanced growth

$$
\max \left(\sqrt{\frac{A_{\ell}(\sigma)}{A_{\ell}(0)}}\right)=\left(1-A t^{2}\right)^{-1 / 4} .
$$

Thus, high Atwood number RT growth with contiguous density gradients exhibits the largest deviations from classical growth at the lowest mode numbers. This conclusion is not particularly dire since RT growth is generally secular in spherical systems - not exponential - unless the trajectory of the interface varies exponentially with time [30]. The transition from spherical to planar geometry for a given mode depends on the change in effective wavelength $2 \pi R / \ell$ with spherical radius. A nearly constant perturbation wavelength occurs if $\ell>>2 \pi$, leading to near exponential growth [See Eq. (41)]. Thus, mode numbers between 50 and 100 roughly define the transition to planar behavior.

In the planar limit, RT growth is generally described by Eq. (42). Figure (6) illustrates the growth for the simpler but still useful case: $\sigma_{1}=\sigma_{2} \equiv \sigma$ [See Eq. (1b)]. A maximum occurs in the RT growth rate spectrum at a wavenumber $k_{c}$ such that:

$$
k_{c} L_{\rho}=\frac{\sqrt{1-A t^{2}}}{2 A t},
$$

with the maximum growth rate given by Eq. (56b). A maximum occurs for $L_{\rho}>0$ because the co-alignment of the density gradients supports an increase in the effective Atwood number of a perturbation. At longer wavelengths density-gradient stabilization 
sets in to depress RT growth. In the regime $1-A t<<2 k^{2} L_{\rho}^{2} \ll 1$ Eq. (1b) reduces to: $\gamma_{R T}=\sqrt{g / L_{\rho}}$, which interestingly enough coincides with the expression for densitygradient stabilization from a continuous density profile in the short wavelength limit, cf., Eq. (1a). For yet larger wavelengths such that $2 k^{2} L_{\rho}^{2} \ll 1-A t$ we obtain: $\gamma_{R T}=k \sqrt{2 A t \cdot L_{\rho} g /(1-A t)}$ which vanished with $k$.

For impulsive acceleration, i.e., $g(t)=\Delta v_{0} \cdot \delta\left(t-t_{0}\right)$, Eq. (42) applies as well to the description of RM growth [20]. Integrating Eq. (41) yields for the RM growth rate:

$$
\gamma_{R M} \equiv \frac{d \ln a_{k}}{d t}=\frac{A_{k} k \cdot \Delta v_{0}}{1+A_{k} k \cdot \Delta v_{0} \cdot\left(t-t_{0}\right)}
$$

where $d a_{k}\left(t<t_{0}\right) / d t=0$. By virtue of the linear dependence of RM growth rate on $A_{k}$ at early times compared with the RT growth rate $\left(\propto \sqrt{A_{k}}\right)$, contiguous density-gradient effects can be comparatively larger. For $L_{\rho}>0$ increased growth results according to:

$$
\gamma_{R M}=\frac{A t \cdot k \cdot \Delta v_{0}}{1+A t \cdot k \cdot \Delta v_{0} \cdot\left(t-t_{0}\right) \cdot\left(\frac{2 k L_{\rho}}{\sqrt{1+4 k^{2} L_{\rho}^{2}}-A t}\right)} \cdot\left(\frac{2 k L_{\rho}}{\sqrt{1+4 k^{2} L_{\rho}^{2}}-A t}\right) .
$$

Finally we note that for first-shock passage across the interface, Eq. (5) or Eq. (8c) for $L_{\rho}$ may not apply and other arguments are required. For example, x-ray preheat of a low-Z shell can lead to volumetric expansion of the shell and the initial establishment of contiguous density gradients on the inner interface well before shock transit. It must also be mentioned that Eq. (59) is valid only in the limit of weak shocks, lest our model assumptions of small $\delta \rho_{i} / \rho_{i}$ become violated [See below Eq. (12)].

\section{Application to an ICF implosion}


We apply the model to a particular example of an Omega-scale ICF implosion briefly mentioned in Section IIa [See Fig. (2c)]. A challenging exercise towards the goal of understanding the hydrodynamic stability of an imploding shell is carrying out a suite of 2-D single-mode growth-factor calculations. To maintain perturbation growth in the linear regime an infinitesimally small, single-mode perturbation amplitude is imposed. A suite of simulations over a range of mode numbers is undertaken to generate a spectrum of growth factors on a chosen interface at a given time - usually taken as the instant of peak neutron production. This spectrum is then convolved with an initial surface spectrum and summed in quadrature to estimate the annular extent of interface mix.

In practice the generation of a linear growth-factor spectrum is time-consuming and fraught with numerical challenges in controlling mesh instabilities. Various numerical filters are often employed to suppress such instabilities, but at the risk of possibly compromising the integrity of the calculation. An alternative approach is the combined use of analytical models as developed in this work with 1-D simulations to estimate 2-D growth factors. For example, the various time-dependent outputs from a highly resolved 1-D simulation of the imploding shell can be directly used in the governing equation for perturbation growth, Eq. (30).

It is worth noting that an important physical effect not explicitly included in our Plessettype analysis is the diffusion of thermal energy along a perturbed interface. This feature is particularly important for short wavelength perturbations where classical RayleighTaylor growth can be drastically moderated. Bychkov et al. [31] have derived an appropriate expression for ablatively-driven RT growth valid in the limit of short wavelengths compared with the temperature gradient scalelength $\left(k L_{T}>>1\right)$ :

$$
\gamma_{R T}=\sqrt{k g+\frac{k^{4} \chi^{2}}{4}}-\frac{k^{2} \chi}{2},
$$

where $\chi \equiv \kappa / \rho c_{p}$ is the thermal diffusivity and $\kappa$ is the thermal conductivity. A similar behavior is well known for the case of momentum diffusion or ion viscosity [32]. 
However, thermal diffusivity typically dominates over viscosity since electron transport is far faster than ion transport [33]. For the longer wavelengths of interest in this work the effects of thermal diffusion are relatively modest in practice. Still, it is useful to try to consider its effect in the present analysis for comparison with 2-D growth-factor simulations that generally include thermal transport.

Although the current treatment as it stands does not include thermal transport, phenomenological modifications to include the short-wavelenth behavior depicted in Eq. (60) can be carried through. First, we assert that the gradient-temperature scalelength is on the order of a density-gradient scalelength. This assertion follows from an assumed ideal equation-of-state [See Eq. (8b)] and the fact that RT growth begins at deceleration onset: $\vec{\nabla} P=0$. Second, we trigger the effects of thermal diffusivity by establishing a hard cutoff in $k L_{T}$ above which thermal transport will be included in the 1-D calculation in a form similar to Eq. (60):

$$
k L_{T}>2 \cdot\left(\frac{\ell}{R}\right) \cdot\left(\frac{L_{\rho 1}+L_{\rho 2}}{2}\right)=\ell \cdot\left(\frac{1}{\sigma_{1}}+\frac{1}{\sigma_{2}}\right) .
$$

Equation (61) is an attempt to satisfy the requirement for validity of Eq. (60) $\left[k L_{T}>>1\right]$ while employing an average of $L_{T i}$ across the classical interface. Likewise, the effective thermal diffusivity for the interface is appropriately written as a sum: $\chi=\chi_{1}+\chi_{2}$.

In the example under consideration we will focus on RT growth following deceleration onset. The influence of RM growth is often small compared with RT, and we choose to ignore the effects of thermal transport during the earlier episodes of RM growth in an ICF implosion. The reason for this choice is that the influence of thermal transport on RM growth has not been worked out to our knowledge, although asymptotic studies of the Navier-Stokes equation have succeeded in including the related effects of viscosity [34]. A further assumption is that the terms proportional to $\sigma_{i}$ and $d \sigma_{i} / d t$ be dropped in Eq. (30) prior to deceleration onset. The reason for this simplification is that the characteristically strong shocks found in an ICF implosion lead to large density 
perturbations and exceedingly small density-gradient scalelengths which violate the underlying assumptions of the model: $\varepsilon_{i} \ll<1$ [See Section IIC].

Figure 7 shows the predicted 2-D growth factors up to peak neutron production according to our 1-D model (with and without thermal transport) and compared with the results of detailed 2-D single-mode simulations. For this exercise we have assumed equal polytropes $\left(\gamma_{1}=\gamma_{2}\right)$ in applying the analytical model [Eq. (30)]. The points that are labeled 1-D with $\chi, \sigma=0$ represent classical RT growth including BP growth as obtained from directly integrating Eq. (30). Compared with the 2-D simulation results, a conventional treatment of RT and BP growth is seen to significantly underestimate the growth of the lowest-order modes. In this regime the results are rather insensitive to thermal diffusivity and its manner of implementation [Eq. (61)]. Beyond $\ell \approx 20$ the classical description deviates greatly from the 2-D results, strongly suggesting that thermal transport is critically important. The points labeled 1-D with finite $\chi, \sigma$ now include all terms in Eq. (30) following deceleration onset - though with one exception. We have taken $\alpha=2$ [See Eq. (32)] for the imploding shell in order to avoid large density changes in time that invalidate the model assumptions as discussed above. This assumption follows from applying mass conservation to an imploding, fully compressible shell and is consistent with previously reported numerical results [12].

Figure (7) shows that the 1-D model complete with density-gradient and thermal effects tracks the 2-D growth-factor spectrum fairly well. Beyond $\ell \approx 10$ some sensitivity to the way in which thermal transport has been implemented is found, particularly for the intermediate mode numbers $(\ell \approx 30)$. A first-principles analysis that includes thermal transport in a spherical geometry would overcome this element of arbitrariness in the current model. However, the main focus and interest of this work resides in the lowestorder modes which are largely immune to thermal transport.

\section{Summary}


A Plesset-type analysis has been applied to the study of the effects of contiguous density gradients on an accelerating, spherical, classical interface. In contrast to previous work with smoothly varying density profiles on an unstable interface, reduced RT growth on a classical interface with contiguous density gradients is predicted to occur for long wavelength perturbations. Intermediate wavelength perturbations are shown to exhibit enhanced growth compared with classical RT due to an effective increase in the Atwood number $(A t>0)$ from a co-alignment of the contiguous density gradients $\left(L_{\rho}>0\right)$ with the acceleration $(g>0)$. The short wavelength limit is shown to recover classical RT growth. A convenient analytic expression describing RT growth for all wavelengths in the planar limit is given by Eq. (1b).

The effects of contiguous density gradients on classical BP growth are shown to be generally modest but destabilizing for ICF-relevant conditions. A new pathway for geometric growth is predicted for an accelerating spherical shell in the presence of strong and contiguous density gradients $(\sigma>>1)$. This instability is found to occur only for the lowest order modes and is independent of the classical Atwood number $(A t)$.

A linearized analysis of Bernoulli's equation has shown a potential resonance condition between the interface radius $R$ and the interior fluid density-gradient scalelength $L_{\rho, 1}$, provided the converging (fuel-pusher) interface undergoes acceleration $(g<0)$. This resonance cannot occur during RT instability growth that follows deceleration onset, although RM growth may be enhanced in the neighborhood of the resonance condition prior to stagnation - but only when the adiabatic indices are distinct $\left(\gamma_{1} \neq \gamma_{2}\right)$.

The analysis has made a physical distinction between compressible and incompressible perturbation response in the presence of contiguous density gradients despite satisfying the standard mathematical requirement for compressibility: $\nabla^{2} \delta \Psi \neq 0$. We have argued that the physical criterion for compressible perturbation behavior is whether the associated density response can occur over a representative timescale of instability growth. In this sense, the boundary mode number for compressible response will be a function of the instability in question. We have shown that the RT instability with 
contiguous density gradient profiles generally behaves in an incompressible manner for conditions of interest in most ICF applications.

A comparison of the 1-D analytic model [Eq. (30)] with detailed 2-D single-mode growth-factor simulations of an Omega-class ICF implosion [19] has been carried out. The anomalously high perturbation growth of the higher-order modes shows clearly the need to implement thermal transport in the current analytical model. A heuristic attempt to include thermal diffusivity is largely successful at suppressing classical RT growth at high mode numbers. The lowest-order modes are unaffected by thermal diffusivity and are well described by the analytic model according to direct comparisons with the 2-D single-mode growth-factor simulations. The focus of this work in spherical geometry has been to understand the growth of low-order modes where BP effects are expected to be important and thermal transport plays essentially no role. Still, the formal inclusion of thermal transport in our Plesset-type treatment should help to redress the current deficiency at high mode numbers.

\section{Acknowledgments}

Useful discussions with Karnig Mikaelian, Jose Milovich, Harry Robey and Sasha Velikovich are gratefully acknowledged. This work was performed under the auspices of the U.S. Department of Energy by the University of California Lawrence Livermore National Laboratory under Contract No. W-7405-Eng-48.

\section{Figures}

Fig. 1: Schematic of ICF geometry showing generic mass density $(\rho)$ profiles versus spherical radius coordinate $(r)$ with density discontinuity at interface $r=R$.

Fig. 2a-c (color): (a) Density profiles versus radius normalized to minimum fuel radius in fuel (solid line) and $\mathrm{Au}$ (thick solid line) at deceleration onset $\left(t=t_{D}\right)$ and indicated 
later times for ignition double-shell design [Ref. 11]; (b) $\sigma_{i}$ versus time for ignition double-shell design from deceleration onset to time of minimum fuel radius; (c) densitygradient scalelengths versus time for Omega-class, hohlraum-driven, single-shell implosion [See Ref. 19] after deceleration onset on fuel-side of interface (red) and pusher-side of interface (green) .

Fig. 3 (color): Schematic of (a) incompressible and (b) compressible perturbed density response to modulated interface.

Fig. 4 (color): Normalized $\beta$-factor for BP growth [Eq. (45)] versus Legendre mode number and dimensionless gradient-scalelength parameter $\sigma$ for density mismatch $\rho_{1} / \rho_{2}=0.3$ and shell compressibility parameter $\alpha=2$.

Fig. 5a-b (color): (a) Normalized RT growth rate versus Legendre mode number and dimensionless gradient-scalelength parameter $\sigma$ for $\rho_{1} / \rho_{2}=0.3$ and shell compressibility parameter $\alpha=2$; (b) versus Legendre mode number and $\rho_{1} / \rho_{2}$ for $\sigma=50$.

Fig. 6 (color): Planar limit of RT growth rate [See Eq. (1b)] normalized to classical RT versus $k L_{\rho}$ and Atwood number At for $\sigma_{1}=\sigma_{2} \equiv \sigma$. Solid lines denote $L_{\rho}>0$; dotted lines correspond to $L_{\rho}<0$.

Fig. 7 (color): Simulated perturbation growth factors versus Legendre mode number an Omega-scale implosion [See Fig. (2c)] from 2-D single-mode simulations (green), 1-D simulations based on Eq. (30) without density-gradient $\sigma$ effects and thermal diffusivity $\chi$ (red), and 1-D simulations based on Eq. (30) including finite $\sigma, \chi$ effects (blue). Open red squares denote rescaled growth factors at higher mode numbers.

\section{References}


[1] S. Atzeni and J. Meyer-ter-Vehn, The Physics of Inertial Fusion (Oxford University Press, Oxford, 2004).

[2] J.D. Lindl, Inertial Confinement Fusion (Springer-Verlag, New York, 1998); J.D. Lindl, P. Amendt, R.L. Berger et al., Phys. Plasmas 11(2), 339 (2004).

[3] J.M. Soures, R.L. McCrory, C.P. Verdon et al., Phys. Plasmas 3, 2108 (1996).

[4] K.O. Mikaelian, Phys. Rev. Lett. 48 (19), 1365 (1982).

[5] J.D. Lindl, "Hydrodynamic and plasma stability limitations on the choice of laser wavelength for radiation-driven ICF implosions", Laser Program Annual Report (1983), Lawrence Livermore National Laboratory, Livermore, CA, UCRL-50055-83, pp. 2-40 to 2-46 (unpublished). Copies may be obtained from the National Technical Information Service, Springfield, VA 22161.

[6] D.H. Munro, Phys. Rev. A 38 (3), 1433 (1988).

[7] A.B. Bud'ko and M.A. Liberman, Phys. Fluids B 4 (11), 3499 (1992).

[8] R. Betti, V.N. Goncharov, R.L. McCrory and C.P. Verdon, Phys. Plasmas 5, 1446 (1998).

[9] S. Fujioka, A. Sunahara, K. Nishihara et al., Phys. Rev. Lett. 92 (19), 195001 (2004).

[10] S. Fujioka, H. Shiraga, M. Nishikino et al., Phys. Plasmas 10 (12), 4784 (2003).

[11] S.A. Colgate and A.G. Petschek, "Minimum conditions for the ignition of fusion", LA-UR-88-1268, 1988; copies may be obtained from the National Technical Information Service, Springfield, VA 22161; D.B. Harris and W.S. Varnum, Bull. Am. Phys. Soc. 41, 1479 (1996); P. Amendt, J.D. Colvin, R.E. Tipton et al., Phys. Plasmas 9 (5), 2221 
(2002); J.L. Milovich, P. Amendt, M. Marinak, and H. Robey, Phys. Plasmas 11 (3), 1552 (2004).

[12] P. Amendt, J.D. Colvin, J.D. Ramshaw, H.F. Robey, and O.L. Landen, Phys. Plasmas 10 (3), 820 (2003).

[13] M.E. Glinsky, D.S. Bailey, R.A. London, P.A. Amendt, A.M. Rubenchik, and M. Strauss, Phys. Fluids 13, 20 (2001).

[14] S. Higenfeldt, D. Lohse, and M.P. Brenner, Phys. Fluids 8, 2808 (1996); H. Lin, B.D. Storey and A.J. Szeri, Phys. Plasmas 7 (12), 5118 (2000); H. Lin, B.D. Storey and A.J. Szeri, J. Fluid Mech. 452, 145 (2002).

[15] G.I. Bell, Los Alamos Scientific Laboratory Report No. LA-1321, 1951; M.S. Plesset, J. Appl. Phys. 25, 96 (1954).

[16] I.B. Bernstein and D.L. Book, Phys. Fluids 26 (2), 453 (1983).

[17] A.M. Lezzi and A. Prosperetti, Phys. Fluids A 1 (11), 1784 (1989).

[18] W.L. Kruer, Physics of Laser Plasma Interactions (Addison-Wesley, Redwood City, CA, 1988).

[19] P. Amendt, R.E. Turner, and O.L. Landen, Phys. Rev. Lett. 89, 165001 (2002).

[20] K.O. Mikaelian, Phys. Fluids 17, 034101 (2005).

[21] J.D. Ramshaw and P.A. Amendt, Phys. Rev. E 67, 056304 (2003).

[22] S.W. Haan, Phys. Rev. A 39 (11), 5812 (1989).

[23] R. Epstein, Phys. Plasmas 11 (11), 5114 (2004). 
[24] V.N. Goncharov, J.P. Knauer, P.W. McKenty, P.B. Radha, T.C. Sangster, S. Skupsky, R. Betti, R.L. McCrory and D.D. Meyerhofer, Phys. Plasmas 10, 1906 (2003).

[25] V.N. Goncharov, P. McKenty, S. Skupsky, R. Betti, R.L. McCrory, and C. CherfilsClerouin, Phys. Plasmas 7, 5118 (2000).

[26] H. Lin, B.C. Storey, and A.J. Szeri, Phys. Fluids 14, 2925 (2002).

[27] L.D. Landau and E.M. Lifshitz, Fluid Mechanics (Pergamon Press, $2^{\text {nd }}$ Edition, 1987), p. 4.

[28] D.S. Clark and M. Tabak, Phys. Rev. E 71, 055302(R) (2005).

[29] M.A. Liberman and A.L. Velikovich, Nucl. Fusion 26, 709 (1986).

[30] K.O. Mikaelian, Phys. Rev. Lett. 65 (8), 992 (1990).

[31] V.V. Bychkov, S.M. Golberg, and M.A. Liberman, Phys. Plasmas 1, 2976 (1994).

[32] R.E. Duff, F.H. Harlow, and C.W. Hirt, Phys. Fluids 5, 417 (1962).

[33] D.D. Ryutov, Physics of Plasmas 7, 4797 (2000).

[34] K.O. Mikaelian, Phys. Rev. E 47, 375 (1993); P. Carles and S. Popinet, Phys. Fluids 13, 1833 (2001). 
Fig. 1

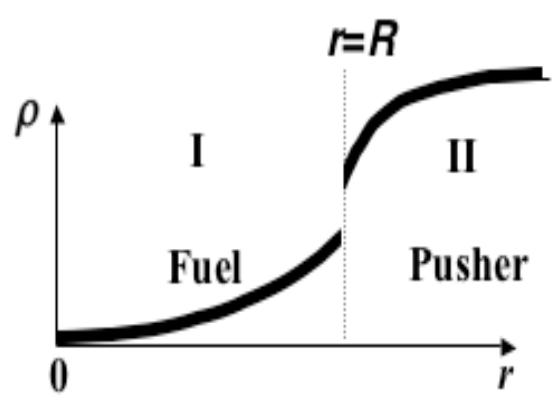


Fig. 2a

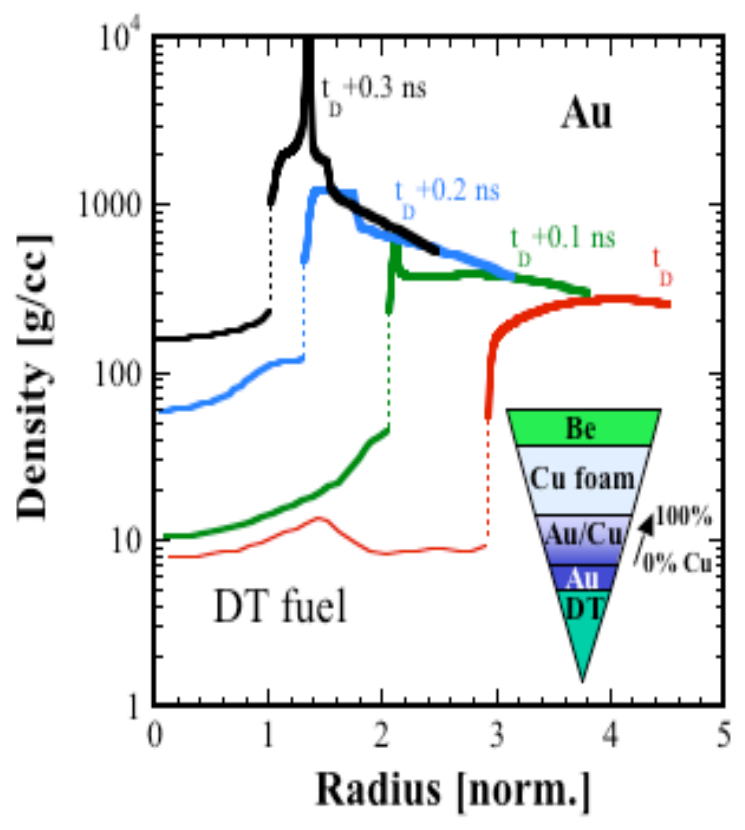


Fig. 2b

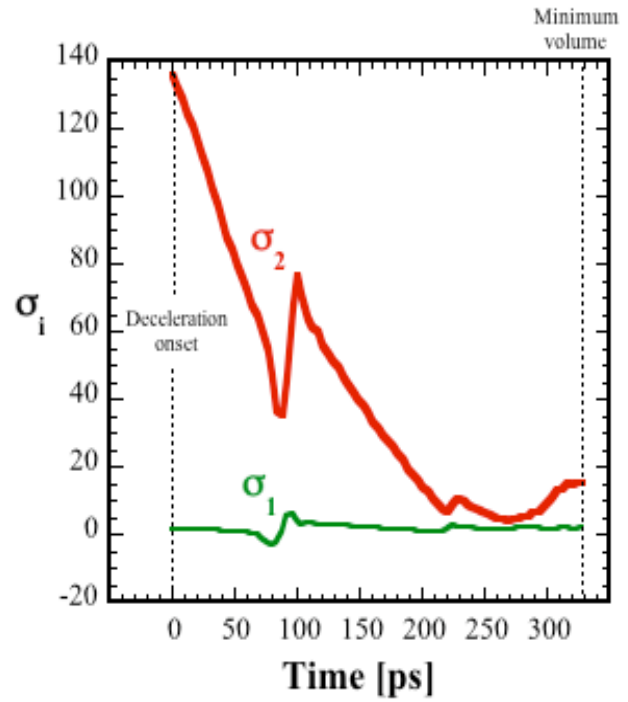


Fig. 2c

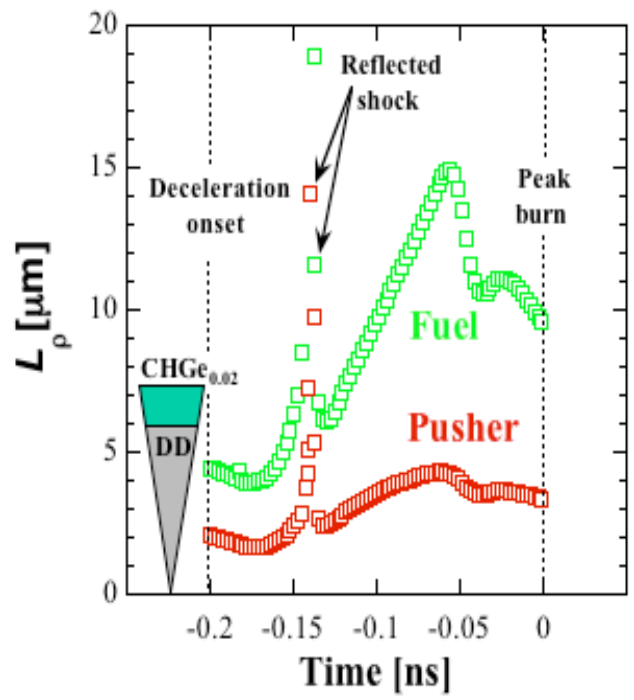


Fig. 3

a)

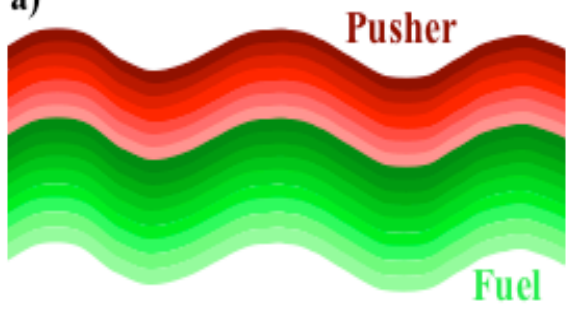

b) Pusher

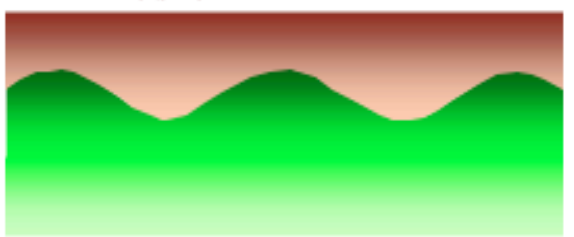

Fuel 
Fig. 4

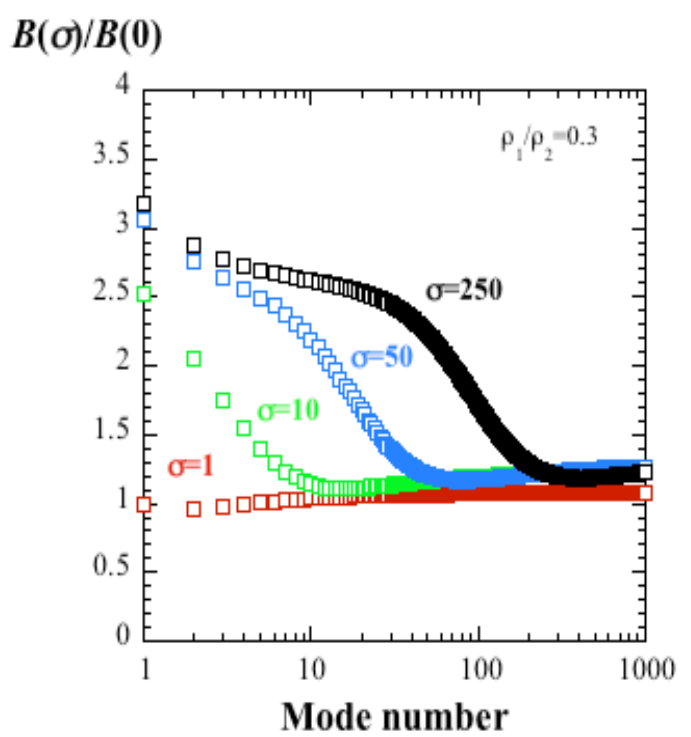


Fig. 5a

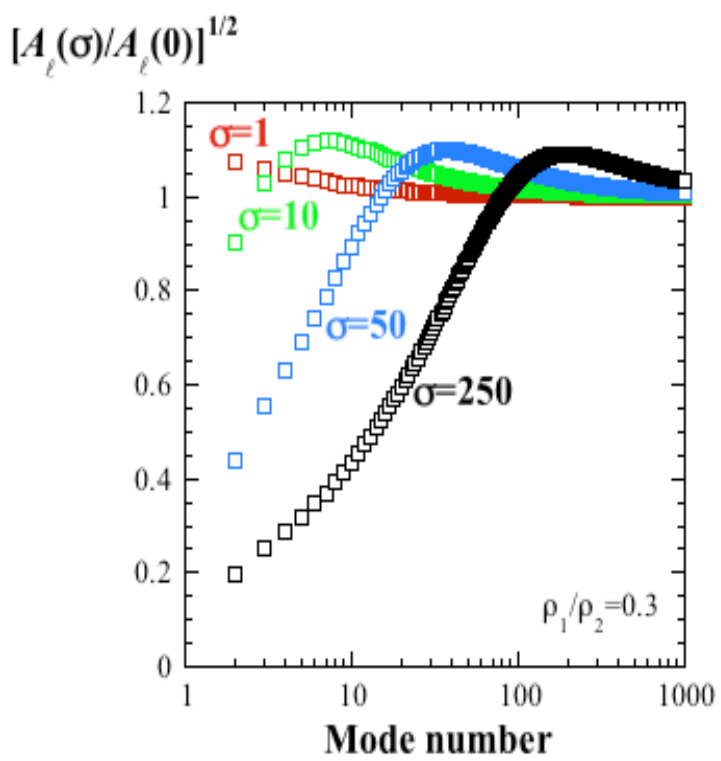


Fig. 5b

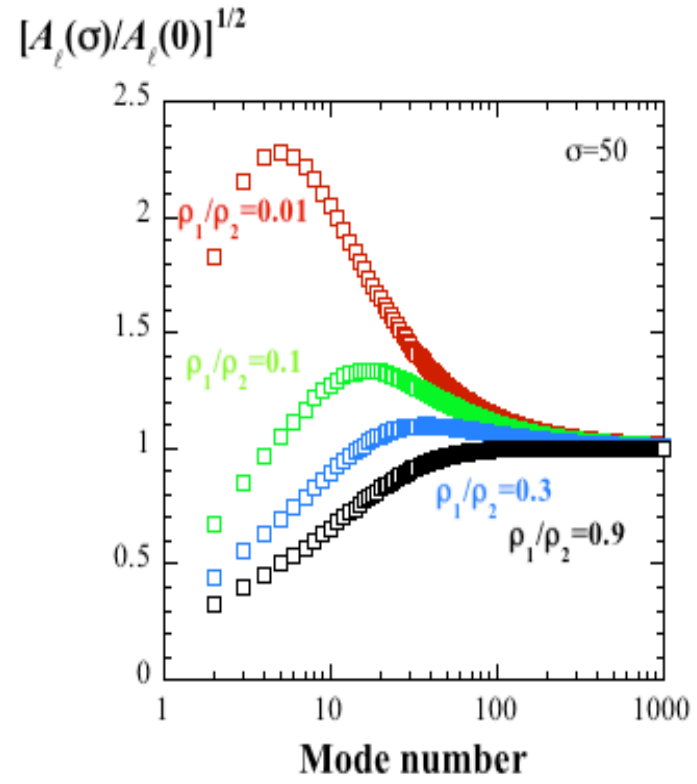


Fig. 6

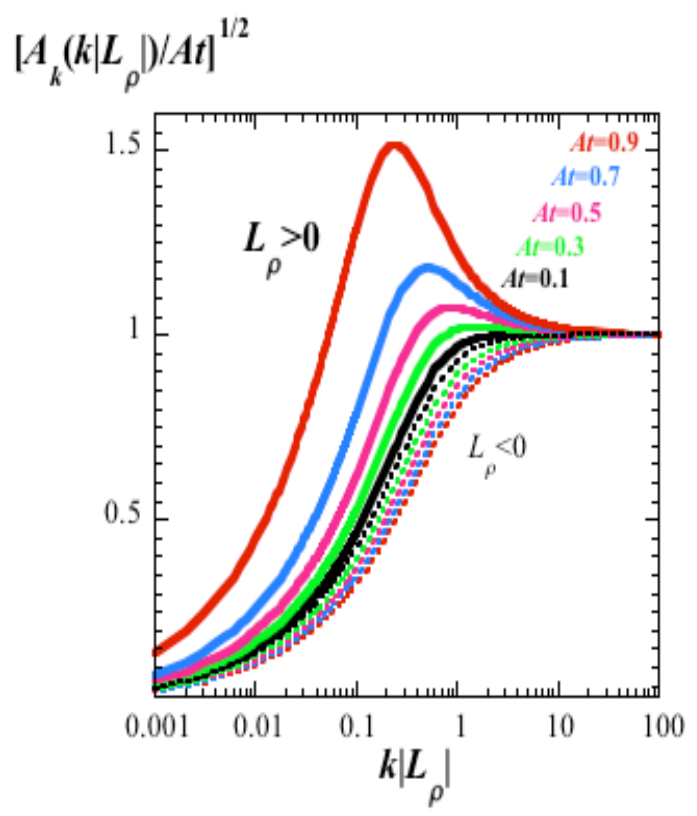


Fig. 7

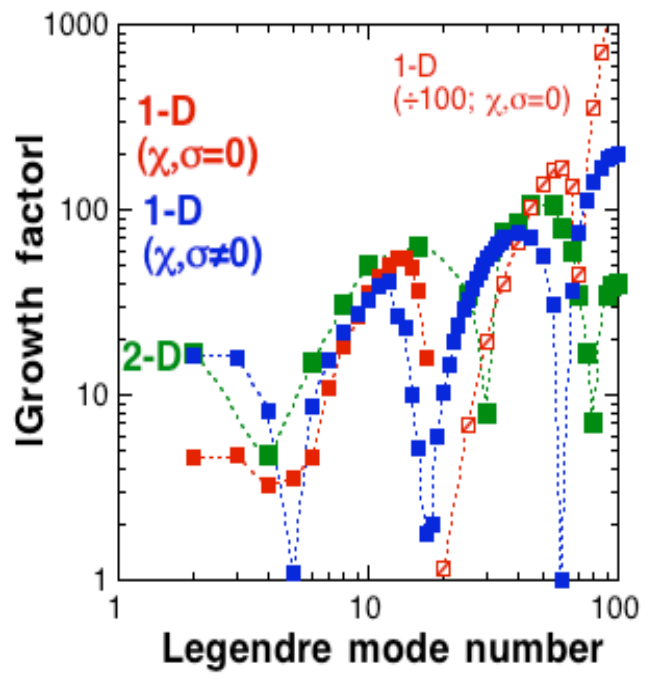

